Neurology

Elsevier Editorial System(tm) for The Lancet

Manuscript Draft

Manuscript Number: THELANCETNEUROLOGY-D-18-00814R2

Title: MRI in traumatic spinal cord injury: from clinical assessment to neuroimaging biomarkers

Article Type: Review

Corresponding Author: Dr. Patrick Freund, Ph.D., M.D.

Corresponding Author's Institution: UCL

First Author: Patrick Freund, Ph.D., M.D.

Order of Authors: Patrick Freund, Ph.D., M.D.; Maryam Seif; Nikolaus Weiskopf; Karl Friston; Michael Fehlings; Alan Thompson; Armin Curt

Abstract: Traumatic spinal cord injury (SCI) occurs when an external physical impact acutely damages the spinal cord and leads to permanent neurologic dysfunction, personal disability and social burden.

Conventional MRI plays a crucial role in the diagnostic workup of SCI patients as it reveals extrinsic compression of the cord and disruption of the discoligamentous complex. Additionally, it can reveal macrostructural evidence of primary intramedullary damage such as haemorrhage, oedema, post-traumatic cystic cavities and tissue bridges. Quantitative MRI (qMRI), such as magnetisation transfer, MR relaxation mapping and diffusion imaging, enables the tracking of secondary changes across the neuraxis at the microstructural level. Both, conventional MRI and GMRI metrics, obtained early after SCI, are predictive of outcome. Thus, neuroimaging biomarkers may serve as surrogate endpoints for more efficient trials targeting acute and chronic SCI. The adoption of neuroimaging biomarkers in SCI centres may eventually lead to individualized patient care approaches. 


\section{MRI in traumatic spinal cord injury: from clinical assessment to neuroimaging biomarkers}

Patrick Freund, $\mathrm{MD}, \mathrm{PhD}^{1,2,3,4}$, Maryam Seif, $\mathrm{PhD}^{1,3}$, Nikolaus Weiskopf, $\mathrm{PhD}^{3,4}$, Karl Friston, $\mathrm{MD}^{4}$, Michael G. Fehlings, $\mathrm{MD}^{5}$, Alan Thompson, $\mathrm{MD}^{2}$ and Armin Curt, $\mathrm{MD}^{1}$

${ }^{1}$ Spinal Cord Injury Center Balgrist, University Hospital Zurich, University of Zurich, Zurich, Switzerland

${ }^{2}$ Department of Brain Repair \& Rehabilitation, UCL Institute of Neurology, UCL, London, UK

${ }^{3}$ Department of Neurophysics, Max Planck Institute for Human Cognitive and Brain Sciences, Leipzig, Germany

${ }^{4}$ Wellcome Centre for Human Neuroimaging, UCL Institute of Neurology, UCL, London, UK

${ }^{5}$ University of Toronto Spine Program and Toronto Western Hospital, Toronto, Ontario, Canada

\section{Corresponding author:}

Prof. Patrick Freund, Spinal Cord Injury Center, Balgrist University Hospital, Forchstrasse 340

8008 Zurich, Switzerland, E-mail: Patrick.Freund@Balgrist.ch, Phone: +41 443861111 


\begin{abstract}
Traumatic spinal cord injury (SCI) occurs when an external physical impact acutely damages the spinal cord and leads to permanent neurologic dysfunction, personal disability and social burden. Conventional MRI plays a crucial role in the diagnostic workup of SCI patients as it reveals extrinsic compression of the cord and disruption of the discoligamentous complex. Additionally, it can reveal macrostructural evidence of primary intramedullary damage such as haemorrhage, oedema, post-traumatic cystic cavities and tissue bridges. Quantitative MRI (qMRI), such as magnetisation transfer, MR relaxation mapping and diffusion imaging, enables the tracking of secondary changes across the neuraxis at the microstructural level. Both, conventional MRI and qMRI metrics, obtained early after SCI, are predictive of outcome. Thus, neuroimaging biomarkers may serve as surrogate endpoints for more efficient trials targeting acute and chronic SCI. The adoption of neuroimaging biomarkers in SCI centres may eventually lead to individualized patient care approaches.
\end{abstract}




\section{Introduction}

Conventional MRI of the spinal cord is an essential component in the diagnostic investigation, surgical treatment, and rehabilitation of patients with spinal cord injury (SCI). ${ }^{1}$ Spinal MRI is the gold standard for the evaluation of any damage to the disco-ligamentous complex (i.e. spine instability and spinal canal encroachment) and neural structures (i.e. integrity of the spinal cord) induced by mechanical trauma. ${ }^{2}$ In clinical practice sagittal and axial T2-weighted MRI sequences are usually applied and can be complemented with a shortT1 inversion recovery (STIR) sequence. ${ }^{3}$ These conventional MRI sequences reveal the level of the damage and the extent of intra/extramedullary abnormalities (oedema and haemorrhage), the degree of spinal cord compression, extent of disk herniation, and ligamentous instability at the level of the injury. ${ }^{3}$ Coupled with the clinical examination, these imaging findings obtained within hours of the trauma, guide decision making and lead to a timely and appropriate decompression of the contused and compressed spinal cord. ${ }^{4}$

Despite their critical importance in clinical management, these conventional MRI sequences provide relatively less information about the evolving microstructural changes of the immediate and adjacent spinal cord segments and, subsequently, of the brain. There is a pressing need for a more in-depth understanding of both the complex processes of neural plasticity, at the microstructural level, and the complex functional interactions between spinal and supraspinal networks involved in SCI recovery. ${ }^{5}$ Such information can help us to understand the pathobiology as it enables the tracking of neuronal changes at the microstructural level across the neuraxis. Spinal imaging studies from a number of centres have employed advanced quantitative MRI (qMRI) techniques, such as magnetisation transfer, MR relaxation mapping, and diffusion imaging to improve detection and quantification of microstructural features of trauma-induced pathology both at and remotely 
from the site of injury. ${ }^{6-14}$ These qMRI protocols provide quantitative measures of spinal cord $^{15,16}$ and brain integrity ${ }^{17}$ that reflect atrophy, demyelination, and iron deposition of tissue. They have been used to demonstrate widespread $^{6-14}$ and progressive neurodegeneration $;^{7-9}$ the magnitude of which predicts clinical recovery. ${ }^{9,10}$ qMRI therefore offers improved assessments of underlying neural integrity and can provide insights into the relationship between clinical recovery and neural plasticity, within the spinal cord and the brain. ${ }^{18}$ Additionally, task-based (fMRI) and resting state (rs-fMRI) functional MRI, although non-quantitative, can probe plasticity at the level of the brain, ${ }^{19-21}$ and within the spinal cord. $^{22-25}$ In clinical practice, sensorimotor impairments assessed by means of the International Standards for Neurological Classification of Spinal Cord Injury (ISNCSCI) ${ }^{26}$ are commonly used as predictors of outcome following SCI. Now conventional MRI markers such as the Basic Score ${ }^{27}$ and intramedullary lesion length (IMLL) ${ }^{28}$ are considered as useful predictors of outcome. However, the future portends a better understanding of traumainduced microstructural changes by means of qMRI (e.g. magnetization transfer, relaxation maps and diffusion characteristics) and a potential use of qMRI as an indicator of outcome in clinical trials.

In this review, we evaluate findings from conventional MRI and discuss the insights they have provided concerning the primary pathological features of the injury epicentre. We then assess developments in qMRI imaging studies that have shone new light on secondary pathological changes affecting the entire neuraxis. The relevance and implications of these advances for improving the ability to predict recovery are discussed, followed by an assessment of their application as biomarkers in trials of patients with acute ( and chronic (>6 months) SCI. Studies assessing cortical and spinal functional plasticity by means of fMRI and rs-fMRI are reviewed before providing recommendations concerning the application of 
MRI protocols in clinical and research settings. Finally, we suggest directions for future research.

\section{Conventional MRI}

\section{Immediate changes at the epicentre}

The majority of SCI patients undergo decompression surgery and receive spinal fixation devices (i.e. metallic implants) to manage spinal instability. The presence of metallic implants causes significant MRI artifacts such as signal-loss, signal-pileup, geometric distortion, and failure of fat suppression, ${ }^{29}$ which worsen with increasing magnetic field strength. ${ }^{30}$ These image artefacts limit MRI diagnostic utility and reduce the quality of the qMRI metrics. Current strategies for metal artefact suppression, that allow scan acquisitions in a clinically feasible duration, include the slice-encoding for metal artefact correction ${ }^{31}$ with dual-source parallel radiofrequency, ${ }^{32}$ as well as compressed-sensing multi-spectral imaging techniques. ${ }^{33}$ By taking advantage of such techniques, spinal cord imaging studies have investigated primary changes (i.e. macrostructural) immediately following the injury, focally at the injury site and based on hyperintensity signal changes of sagittal and axial T2-weighted and hypointensity signal changes of T1-weighted MRI scans. ${ }^{28,34-39}$ The most prominent features on sagittal T2-weighted scans include haemorrhage, cytotoxic oedema, and spinal cord swelling. ${ }^{28,37}$ Serial quantification of sagittal T2-weighted hyperintensity revealed that the intramedullary damage dynamically expands rostrally and caudally, with injury severity substantially affecting the rate of expansion. ${ }^{27,28,37}$ Based on T2-weighted signal abnormalities, a 5-point ordinal MRI score referred to as the BASIC score has been proposed for MRI-based diagnostic and prognostic classification in patients with acute SCI. ${ }^{27}$ The BASIC score quantifies five distinct patterns of intramedullary T2-weighted signal abnormality in the axial plane at the injury epicentre of the spinal cord (Figure 1). These 
patterns range from no abnormalities to the most severe abnormalities consisting of mixed haemorrhage and oedema. The feasibility and prognostic validity of BASIC scores have been demonstrated both for patients with acute cervical ${ }^{27,40}$ and thoracic ${ }^{41}$ SCI, where MRI had been performed within days after injury. Moreover, the intramedullary lesion size, measured on sagittal T2-weighted slices (Figure $2 \mathrm{~A}$ ), is a good predictor of recovery as its size is influenced by injury severity ${ }^{34-38}$ and the outcome of surgical decompression. ${ }^{28}$

A caveat of quantifying intramedullary damage using conventional MRI scans is the nonspecificity of T2-weigthed signal changes to the underlying pathophysiology. T2-weighted signal changes may reflect various processes, including oedema, inflammation, and the development of myelomalacia, demyelination, or cyst cavitation. ${ }^{27}$ Moreover, interpretation should be dependent on the timing of MRI assessments as the evolution of oedema and haemorrhage changes considerably and is highly variable across patients. ${ }^{28}$ Finally, the quantification of changes in T2-weigthed MRI is usually performed manually by a user with experience in processing conventional MRI, as fully automated methods that can reliably distinguish artefact-induced signal changes at the epicentre of a traumatic lesion are currently absent. Thus, the utility of the BASIC score, as well as the quantification of the intramedullary lesion length, requires further validation. Multicentre studies, both at early and later time points would be ideal, for example, during rehabilitation where the T2weighted signal abnormalities have evolved. ${ }^{42}$

\section{Evolution of changes at the epicentre}

A longitudinal study of thirteen SCI patients with cervical injury employing conventional MRI has investigated the natural sequelae of macrostructural intramedullary changes at the focal injury site during the first year post-SCI ${ }^{42}$. T2-weigthed scans showed a transition from 
the acute oedema and haemorrhage ${ }^{43}$ to sub-acute intramedullary lesion expansion ${ }^{34,37}$ (Figure 3). After signs of oedema and haemorrhage slowly evolved, a post-traumatic cyst appeared in all of thirteen patients within the first month post-SCI. ${ }^{42}$ At this stage it is possible to detect small tissue bridges around the post-traumatic cyst that can be measured at the dorsal and ventral aspect of the cord, adjacent to the cyst on midsagittal T2-weighted scans (Figure 2 B-D). ${ }^{42,44}$ Crucially, the width and location of these tissue bridges predict tract-specific electrophysiological information flow ${ }^{42}$ and long-term functional recovery. ${ }^{42,44}$ Thus, the quantification of spared midsagittal tissue bridges on T2-weighted scans, at 1month post-SCI, holds potential as an important prognostic tool.

The ability of MRI markers of lesion characteristics as well as tissue bridges emphasise the importance of currently conventional MRI protocols to be applied in the clinical setting. ${ }^{45}$ In particular, T2-based scans can detect dynamic intramedullary signal changes as well as preserved midsagittal tissue bridges. ${ }^{42}$ Both can serve as an important diagnostic and prognostic tool, being sensitive to therapeutic interventions. Conventional MRI protocols are also easily applied in longitudinal designs at any stage of SCI and thus could furnish neuroimaging biomarkers for clinical trials. ${ }^{42}$

\section{Progressive cord atrophy}

Despite these insights provided by manually quantifying the primary effects of the trauma at the epicentre of the injury, automated and unbiased quantification of trauma-induced changes at the level of the injury are still not feasible largely due to the artefacts induced by metal implants at the lesion site. One strategy to measure structural changes free of metal artefacts and hence capable of being performed fully or semi-automatically - is to target the artefact free spinal cord above and below the level of injury. A prospective, longitudinal MRI investigation of fifteen traumatic SCI patients, tracking changes to the cross-sectional cord 
area (measured in $\mathrm{mm}^{2}$ ) based on a T1-weigthed MPRAGE sequence at the cervical cord level (C2/C3), showed signs of remote spinal cord atrophy within two months of the SCI. ${ }^{8,46}$ Over time (one year post SCI), atrophy continues to progress, ${ }^{7,8}$ at the level of the cervical cord, reaching $14 \%$ reduced cross-sectional cord area compared with healthy controls in the chronic phase post-SCI. ${ }^{6,9}$ Interestingly, the rate of spinal cord atrophy only showed signs of deceleration two years later. ${ }^{9}$ In the chronic state of SCI, high resolution multi-echo gradient echo scans, that allow to segment the grey and white matter of the cord, ${ }^{47}$ showed that remote neurodegeneration occurs within the dorsal and ventral horns as well as white matter within the high cervical cord ${ }^{6}$ and lumbar enlargement. ${ }^{48}$ While dorsal horn atrophy at the cervical level was associated with sensory outcome (e.g. ISNCSCI pin-prick scores), ventral horn atrophy was associated with ISNCSCI motor score. ${ }^{6}$ It is still not clear whether the rate of atrophy is related to the lesion level and/or injury severity. ${ }^{7,10,46}$ However, the magnitude of remote spinal cord atrophy within the first six months post-SCI is predictive of functional recovery. ${ }^{9,10,46}$

\section{Progressive brain atrophy}

At the level of the brain, the conventional T1-weighted MPRAGE sequence that covers the brain and cervical cord, has provided insights into remote brain atrophy. Trauma-induced brain atrophy is particularly prominent across the cranial projections of the corticospinal tracts, primary motor cortex, insula, anterior cingulate gyrus, and thalamus..$^{7-9,49-52}$ As in the spinal cord, brain atrophy starts to evolve within the first months after SCI and continues for at least two years post-injury. ${ }^{9,53}$ The resulting changes in tissue volume are clinically relevant. For example, greater volume reductions in the brainstem during the first 6 months post-SCI were associated with poorer recovery of lower limb motor function. Interestingly, performance improvements due to intensive lower limb training in chronic SCI patients lead 
to volume increases within the atrophied brainstem, indicating reorganisation processes. ${ }^{54}$ Likewise neuropathic pain intensity has been shown to be associated with reductions in primary sensory cortices and thalamus ${ }^{49}$ as well as increases in grey matter volume within the anterior cingulate gyrus and primary motor cortices.$^{55}$

qMRI

\section{Advances in MRI technology}

Conventional MRI, although sensitive to macrostructural cord and brain pathology, does not provide specific and quantitative microstructural measures of neurodegeneration and plasticity processes, making it difficult to draw specific conclusions about the underlying cause of the observed signal changes on T1- and T2-weigthed MRI scans. Thus, there is a pressing need to establish the missing link between measured MRI signals and changes in the underlying tissue microstructure and neurovascular function to explain and better understand the disease processes associated with SCI. Novel qMRI protocols of the spinal $\operatorname{cord}^{15,56}$ and brain ${ }^{17,57}$ have the potential to measure neural changes at the microstructural level. This is because the degree of myelination, iron content and neuronal microstructure are reflected in MR relaxation times, magnetisation transfer and diffusion of water molecules which can be measured at the voxel-level in the spinal cord ${ }^{15,56}$ and brain. ${ }^{17,57}$ qMRI aims at providing values comparable between individuals and they are specific to particular structural states, for example axonal degeneration or demyelination. ${ }^{56}$ Key state-of-the-art methods such as relaxometry mapping and diffusion MRI has been identified which may have the potential to reveal the underlying pathophysiology after human SCI. ${ }^{56}$

The most common qMRI technique is diffusion-weighted imaging, which probes the directional diffusivity of water molecules and shows sensitivity and specificity to the axon and myelin pathology. ${ }^{58}$ Frequently, diffusion-imaging data are analysed using a tensor 
model, i.e. applying diffusion tensor imaging (DTI). ${ }^{59}$ However, the tensor model makes several restricting assumptions, which complicate the interpretation of major DTI indices (i.e. fractional anisotropy, axial and radial diffusivity, or mean diffusivity) with respect to the underlying pathology. Novel biophysical models of diffusion contrast are being developed based on different mathematical models and could alleviate this issue, although these modelling approaches have yet to be validated; partially due to acquisition/modelling variability versus biological variability. ${ }^{60}$ The quantitative measurement of relaxation and magnetization transfer parameters has been an area of substantial development, making it more accessible to clinical and preclinical research applications. For example, the multi parametric mapping (MPM) approach combines different MRI modalities in one protocol quantifying MR parameter measures of magnetization transfer (MT), and longitudinal and effective transverse relaxation rates $(\mathrm{R} 1, \mathrm{R} 2 *)^{61}$ (Figure 4). The link between these qMRI metrics and histology has been studied to probe the micro-structure of the human neocortex, focusing specifically on myelin, iron, and neuronal fibre mapping. ${ }^{57}$ MT measures correlate with histologically measured myelin content, ${ }^{62}$ whereas certain quantitative relaxation rate measurements correlate with iron content. ${ }^{63}$ These results may provide useful and specific biomarkers such as oligodendrocyte, glial cells, and iron rich fibres, with potential clinical impact in different pathologies, including SCI. ${ }^{57}$

\section{Clinical qMRI studies}

Building on advances of qMRI methods, studies in patients with SCI have focused on improving the detection and quantification of tissue-specific spinal cord and brain pathology and on elucidating its relationship with clinical impairment. DTI applied to the white matter of the injured spinal cord demonstrates lower fractional anisotropy (FA) (sensitive to myelination, axon diameter, fiber density \& organization) values above and below the lesion, 
both in acute ${ }^{64}$ and chronic patients. ${ }^{6,12}$ For processing diffusion weighted images, the advent of a spinal cord template, ${ }^{65}$ and post-processing tools ${ }^{66,67}$ included in the spinal cord toolbox ${ }^{68}$ now offers the opportunity to assess tract-specific DTI changes at the voxel-level across the entire spinal cord. At both the cervical ${ }^{6}$ and lumbar enlargement ${ }^{48}$ DTI has shown tissue specific decreased fractional and axial diffusivities and increased radial diffusivities in the corticospinal tract and the dorsal columns. The former effects have been associated with axonal degeneration ${ }^{58}$ whereas the latter is associated with demyelination. ${ }^{58}$ The results are suggestive of retrograde and anterograde degeneration of descending motor pathways and ascending afferent spinal projections, respectively. Moreover, the grey matter of the lumbar enlargement featured decreased fractional and axial diffusivity, indicating trans-synaptic degeneration of motor neuron pools deprived of supraspinal input. ${ }^{48}$ DTI applied to the brain showed impaired microstructure along the cranial projection of the corticospinal tract, ${ }^{13,14}$ as well as other brain areas such as the corpus callosum, and fibre tracts such as inferior and superior longitudinal fasciculi, and the inferior fronto-occipital fasciculus; ${ }^{11}$ suggesting largescale structural degeneration and reorganization across the brain.

The MPM protocol ${ }^{61,69}$ (Figure 4), applied to acute SCI patients, revealed that spinal cord atrophy was paralleled by myelin-sensitive MT decreases, ${ }^{7}$ while in brain areas undergoing progressive atrophy, myelin content decreased and iron content increased. ${ }^{7-9}$ For example, the atrophying primary motor cortex showed lower myelin content (reflected by decreased MT and $\mathrm{R} 1^{8}$ ), while the atrophying thalamus showed iron deposition (reflected by increased $\mathrm{R} 2 *^{8}$ ). Moreover, within the cerebellum, accelerating atrophy was paralleled by a deceleration of myelin-sensitive MT. These bidirectional effects suggest the changes in myelination $^{62}$ and iron content, ${ }^{70}$ reflecting dynamic processes in the context of compensation, decompensation and the compounding of functional deficits. ${ }^{5}$ 


\section{Predicting outcome}

Clinical recovery occurs most rapidly within the first six months and plateaus at approximately 2 years post-SCI. ${ }^{71}$ At present, neurorehabilitation is the only known means to improve functional recovery. Neurorehabilitation per se is believed to promote neurological changes such as cortical and spinal cord neural circuit reorganisation, which is assumed to translate into improved function. A few longitudinal qMRI studies within the range of one to two years post-SCI follow-up have found that better ISNCSCI lower extremity motor score recovery was predicted by less cervical spinal cord atrophy, ${ }^{9,10}$ and cord diffusion alterations. $^{72}$ Early after SCI ( $<2$ months post-injury) and at the level of the brain, greater ISNCSCI lower extremity motor recovery was associated with less cranial corticospinal tract atrophy. ${ }^{9}$ At the microstructural level, a worse ISNCSCI pin-prick score was associated with a greater increase in GM R2* in the thalamus, ${ }^{9}$ a better ISNCSCI lower extremity motor recovery was predicted by a smaller decrease in MT in the somatosensory cortex ${ }^{9}$ and a greater decrease $\mathrm{R} 2 *$ in the right cerebellum, ${ }^{10}$ and increased functional connectivity between primary motor cortex and supplementary motor and premotor cortices. ${ }^{46}$ More substantial grey matter atrophy in the cerebellum was associated with impaired light-touch sensation, ${ }^{10}$ while greater increases in neuropathic pain intensity were associated with more extensive microstructural changes (increased R2*) in the secondary sensory cortex, anterior cingulate cortex, and cerebellum. ${ }^{9}$

These longitudinal qMRI studies within a two-year follow-up point to three important and clinically relevant findings: (i) while clinical recovery levels off at two years post-SCI, progressive changes in macroscopic and microstructural markers continue; (ii) while macrostructrual changes slow down at the level of the spinal cord, both macroscopic and microstructural measures of neurodegeneration show sustained changes in the brain; (iii) the changes that have the greatest predictive validity in relation to clinical outcome appear to be 
those at the level of the spinal cord, brainstem and cortex (e.g. spinal cord atrophy, cranial CST atrophy, lower MT in the primary motor cortex) over the first 6 months., ${ }^{9,10}$

\section{Implication for clinical trials}

The primary endpoint of choice in SCI trials so far is an improvement in clinical outcome measures. However, neuroimaging biomarkers have the potential to supplement these clinical measures as they are sensitive to neuronal changes even when they do not yet translate into obvious clinical benefit. Currently, clinical trials employ conventional MRI (e.g. T2weighted signal characteristics of the cord) (Table 1) to account for gross macrostructural changes at the lesion site in the spinal cord for example after stem cell interventions. ${ }^{73-75}$ However, signal intensity changes in conventional MRI do not correspond with the specific and quantitative measures of microstructural deficits (e.g. demyelination and axonal degeneration) (e.g. , XX). ${ }^{15}$ With potential treatments targeting repair of the injured spinal cord, it is imperative to improve clinical trial design and efficiency, optimise patient stratification in the context of disease heterogeneity and identify sensitive trial outcome measures.

Based on the advances in MRI, such as conventional MRI, advanced relaxometry mapping and diffusion MRI, the application of neuroimaging biomarkers for SCI trials, which combine conventional MRI and qMRI assessments, is now feasible. This requires measures sensitive to the earliest changes following injury, which are quantifiable, and which capture neural damage and plasticity. As $\mathrm{qMRI}^{15,57,76}$ is sensitive to microstructural aspects of specific tissue classes of the CNS (e.g. myelin and axonal integrity, and iron concentration), these neuroimaging biomarkers are potentially sensitive to recovery processes and treatment responses. ${ }^{15,17,57}$ Moreover, they bear the potential to provide short term surrogate end-points (i.e. changes over 6-12 months), which may reduce the time and cost associated with novel 
drug development. ${ }^{77,78}$ Despite a therapeutic intervention having an effect on imaging outcome such as halting atrophy, there is still some disconnect between changes in imaging outcomes and clinically meaningful recovery; the ultimate goal of a successful clinical trial. Thus, it may be useful to employ more than one imaging outcome in future trials to maximize understanding and interpretation of clinically meaningful findings.

Deploying advanced qMRI methods in multi-centre trials is challenging however, requiring high quality qMRI techniques such as high field MR scanner (e.g. 3 Tesla), advanced software version and sophisticated image post-processing pipelines to be implemented on the different scanner platforms from different manufacturers and different clinical sites across the globe. Any resulting differences or performance issues may reduce the potential benefits for evaluating new therapies. Moreover, clinical trials usually run over years and hence scanner software and hardware upgrades as well as scanner replacements cannot always be avoided. Thus, there is a need to further improve intra-scanner and inter-scanner comparability of the qMRI protocols. The feasibility of combining multi-centre DTI data has been previously shown in $\mathrm{xx}$ countries using different $3 \mathrm{~T}$ scanner models, software versions and pulse sequences.$^{79,80}$ However, critical parameters such as noise floor level and signal-to-noise floor ratio have to be monitored and adjusted to increase the statistical power estimates. ${ }^{79}$ Likewise, the MPM protocol was validated at 3T MRI scanner for use in multi-centre studies based on custom-made ${ }^{61}$ and manufacturers based ${ }^{81}$ FLASH sequences. Currently, the MPM protocol is being considered for a phase II multi-centre clinical trial (NISCI) (EudraCT: 2016-001227-31) investigating the neutralizing effects of an anti-Nogo-A antibody treatment for SCI. ${ }^{82}$ Thus, there is hope that effect sizes based on qMRI data may afford the opportunity to assess site-specific effects of intervention; essential for the translation of trial efficacy to clinical effectiveness. Hypothetical treatment effects, defined by slower 
longitudinal structural changes in these imaging measures, could be detectable over a realistic timescale (6 months post injury) with potentially lower sample sizes ( $<50$ per treatment arm) than required for traditional clinical readouts. ${ }^{53}$

\section{Task specific and resting state functional MRI}

Much of the discussion above concerned assessment of physical changes in the brain and spinal cord resulting from SCI and during recovery. Just as important is the ability to assess functional reorganization associated with SCI. Functional reorganization can be indirectly quantified, both in the brain and spinal cord, by means of fMRI that tracks task-dependent oxygen consumption that is indirectly related to neuronal activity (e.g. blood oxygen dependent signal (BOLD)). In the absence of an explicit task, neuronal activity can also be studied by means of rs-fMRI analysis, which is based on low frequency spontaneous fluctuations in the BOLD signal. rs-fMRI provides an indirect measurement of connectivity that allows for characterization of distinct functional networks in the brain or spinal cord. ${ }^{83}$

Motor and sensory recovery after SCI is associated with functional reorganization of the sensorimotor networks. ${ }^{84-86}$ fMRI studies after chronic SCI have inferred cortical reorganization through increased task-dependent activation in the primary motor cortex, cerebellum, and parietal lobe. ${ }^{84}$ Interestingly, in 23 complete (AIS A) SCI patients with complete impairment, in clinical terms (AIS A), stimulation below the level of the injury resulted in activation in the relevant somatosensory cortices. ${ }^{86}$ This suggests that preserved tissue bridges ${ }^{42}$ continue to carry functional information, but are insufficient to produce clinically meaningful activations or functions.

Spinal cord fMRI studies have also found that substantial task-related spinal activity, in response to stimuli are retained above and below the injury site. ${ }^{22-24}$ This suggests that the spinal cord is actively engaged in plastic processes that can result in recovery of function. 
Interestingly, this may also contribute to the emergences of neuropathic pain conditions ${ }^{85}$ which has been associated with maladaptive plasticity. ${ }^{49}$ Thus, spinal fMRI is feasible in the clinical setting ${ }^{87}$ and can identify changes in neural processing in relation to the location and extent of injury. Although the fMRI and rs-fMRI are readily available on clinical MR systems with a good spatial resolution, the analysis requires sophisticated post-processing tools ${ }^{68}$ and the interpretation of the functionally activated voxels remains challenging. ${ }^{16}$ Further advances in MRI hardware (sensitive MRI coils ${ }^{88}$ ), and in MRI software (optimized localized shimming ${ }^{89}$ ) and in other areas (eg, image postprocessing) are expected to increase the value of spinal cord fMRI as a biomarker in the near future for probing reorganization and plasticity induced by injury.

The application of rs-fMRI has gained momentum as it does not require an explicit task or active participation of the individual. Both in acute ${ }^{19-21}$ and chronic ${ }^{90-92}$ SCI patients, connectivity changes can be observed across the motor system as well as in areas of cognitive control (i.e. the bilateral dorsal anterior cingulate cortex dACC, dorsal lateral prefrontal cortex (DLPFC) and caudate); with the magnitude of change, in both, relating to the recovery of function. ${ }^{19-21}$ Thus, connectivity changes in brain networks might reflect compensatory strategies to overcome functional deficit. However, rs-fMRI is an emerging field featuring a wide range of pre-processing and analytical approaches, which make it difficult to compare the outcomes of the different studies. Nevertheless, rs-fMRI holds promise as a more reliable and useful outcome measure in the clinical setting. To date - although technically feasible ${ }^{25,83,93}$ there have been no rs-fMRI studies in patients with SCI conducted because it is challenging to obtain reliable rs-fMRI data from the spinal cord in SCI patients due to a number of technical issues; such as the influence of physiological noise and metallic implants. Nonetheless, results obtained in healthy controls have consistently shown robust 
networks with extensive connectivity between spinal cord regions as well as across the brainstem and the spinal cord. ${ }^{83,94}$ Based on these results, rs-fMRI of the injured spinal cord would be expected to demonstrate regions with altered/absent connectivity to other spinal cord regions as well as dynamic connectivity changes during recovery. This would allow to monitor functionally relevant changes within the spinal cord during the process of recovery. This awaits validation.

\section{Conclusions and future directions}

Traumatic SCI causes permanent disability, and yet despite advances in medical management such as improved rehabilitation cares and clinical assessments, many patients are left with substantial neurological impairment. Currently, intensive care measures including blood pressure augmentation, neuroprotective approaches with anti-inflammatories, neurosurgical decompression and stabilization and intensive neurorehabilitation are the only interventions applied to promote partial recovery. ${ }^{71}$ Understanding the pathophysiological sequelae would help to reduce and prevent disease burden and would facilitate the development of effective regenerative and neuroprotective treatments.

Both conventional MRI and qMRI of the spinal cord and brain can guide diagnostic workup, identify predictors of recovery, elucidate SCI pathogenesis and provide surrogate endpoints in future clinical trials. ${ }^{16,45}$ Conventional T2-weighted sagittal and axial MRI are key methods to identify the extent of the intramedullary injury ${ }^{27,28}$ and to identify prognostic parameters such as intramedullary lesion length and preserved midsagittal tissue bridges. ${ }^{37,42,44}$ Advanced qMRI sequences, such as DTI and MPM, applied remotely from the injury can identify microstructural changes such as axonal degeneration, demyelination, and iron deposition across the entire neuraxis ${ }^{15,9}$. Combinations of serial conventional MRI and qMRI represent key modalities for a better assessment of spinal cord function compared to clinical 
assessment, and further, in elucidating the relationship between clinical impairment and remote secondary changes in the spinal cord and brain. In addition, these quantifiable changes appear to have notable predictive validity, rendering them viable outcomes for interventional trials. ${ }^{9,53}$

The advances reviewed in this paper suggest recommend that neuroimaging of the spinal cord should be routinely performed in clinical practice and in interventional trials in a number of instances (Table 2). Conventional MRI should include both sagittal and axial views to assess the level and extent of injury within the first 48 hours (e.g. BASIC score). These scans should be repeated 3-4 weeks later to quantify the dynamics of intramedullary lesion length and to identify the amount of preserved midsagittal tissue bridges. To investigate pathophysiological changes in the research setting, qMRI methods, such as DTI and MPM, should be used as these can probe microstructural changes of the spinal cord and brain. Neuroimaging outcome measures derived from both conventional MRI and qMRI protocols should be considered as they as they are predictors of recovery. ${ }^{9,53}$ However, a careful evaluation of the variance caused by differences between scanners and an assessment of reproducibility is required, adding to the complexity of multicentre trials.

Current understanding of trauma induced changes across the neuraxis remains incomplete (Figure 5). A key requirement to assessing plasticity in vivo is ultra-high spatial resolution on the order of hundreds of microns. To visualize and quantify ultra-scale tissue properties of grey and white matter biophysical models that exploit symmetries in the organization of microstructure are required. Emerging technological and imaging developments at higher field strengths (e.g. 7T MRI scanner), such as improvements in RF coil designs, pulsesequence design, improved localized magnetic field shimming methods, ${ }^{89}$ suppression of 
MRI artefacts induced by orthopaedic implants, ${ }^{33}$ and changes in data sampling schemes ${ }^{56}$ will provide the necessary means for these biophysical models in future research. These models can combine multiple different MRI contrasts with different views on the underlying microstructure to address the intractable problem of accurately making inferences concerning the microstructure from single contrasts. For example, modelling the relative myelination of axons (e.g. g-ratio mapping ${ }^{95,96}$ ). The integration and unifying across the different contrasts and spatial scales (from micrometres to centimetres) is the object of intensive and ongoing research in the MRI community. ${ }^{15,57}$ However, MRI contrasts remain indirect measures of changes in the microstructure and composition of the tissue. Therefore, knowledge about the underlying changes is needed for interpretation of the non-invasive qMRI data and for improving the biophysical models. qMRI data will need to undergo histological validation (cross-validation of the MPM contrasts is currently performed in a multi-national ERANET funded "hMRIofSCI consortium (https://www.neuron-eranet.eu/_media/hMRIofSCI.pdf)) of tissue samples from experimental SCI models in order to shed light on the mechanistic underpinnings of changes observed with different MRI contrasts. Finally, multicentre and longitudinal studies, with large patient cohorts that employ qMRI of the spinal cord and brain would be useful to better characterize primary and secondary disease changes, along with their dynamics, and also support and extent current mono-centric and mainly cross-sectional studies. Increasing our understanding of the sequelae after SCI will allow eventually to predict individual trajectories of recovery.

\section{Acknowledgment}

We thank Dr Joshua Grant for proof reading.

\section{Author's contribution}


$\mathrm{PF}, \mathrm{MS}, \mathrm{NW}, \mathrm{KF}, \mathrm{MF}$, AT and AC contributed to the literature search, data interpretation and writing of the manuscript. PF and MS created the figures and tables.

\section{Search strategy and selection criteria}

We conducted a MEDLINE search focused on traumatic SCI using PubMed including only English language publications from 01.2013 until 01.2019. The search headings included the following words "traumatic spinal cord injury" in combination with search terms "atrophy", “demyelination” “diffusion” conventional MRI”, "quantitative MRI”, “neurodegeneration”, clinical trial", "longitudinal", and "MRI prediction". Further articles were identified by searching the list of references cited in the manuscripts that were reviewed. The final reference list was generated on the basis of relevance to the topics covered in this Review.

\section{Declaration of interests}

Patrick Freund reports grants from ERA-NET NEURON (hMRIofSCI no: 32NE30_173678), grants from NISCI supported by the European Union's Horizon 2020 research and innovation program under the grant agreement No 681094, and supported by the Swiss State Secretariat for Education, Research and Innovation (SERI) under contract number 15.0137, grants from Wings for life charity (No WFL-CH-007/14), grants from International Foundation for Research (IRP-158), during the conduct of the study.

Maryam Seif has nothing to disclose.

Nikolaus Weiskopf reports grants from European Research Council / ERC grant agreement $\mathrm{n}^{\circ}$ 616905, grants from BMBF (01EW1711A B) in the framework of ERA-NET NEURON, grants from BRAINTRAIN European research network (Collaborative Project) supported by 
the European Commission (Grant agreement $n^{\circ}$ 602186), grants from NISCI supported by the European Union's Horizon 2020 research and innovation program under the grant agreement No 681094, and supported by the Swiss State Secretariat for Education, Research and Innovation (SERI) under contract number 15.0137, grants from UCL Impact Awards and Siemens Healthcare, during the conduct of the study; and The Wellcome Centre for Human Neuroimaging and Max Planck Institute for Human Cognitive and Brain Sciences have institutional research agreements with Siemens Healthcare. He is also Associate Editor of Frontiers in Brain Imaging Methods.

Michael Fehlings is chair of the scientific advisory board of Fortuna Fix. MF is supported by grants from the Canadian Institute of Health Research (CIHR), AOSpine, Wings for Life Foundation, Craig Neilsen Foundation and the International Spinal Research Trust (ISRT). He is also supported by the Halbert Chair in Neural Repair and Regeneration and the Dezwirek Foundation.

Karl Friston is funded by a Wellcome Trust Principal Research Fellowship (Ref: 088130/Z/09/Z).

Alan Thompson reports personal fees paid to his institution from Eisai Ltd and fees and support for travel from Hoffmann-La Roche outside the submitted work; and Editorial Board member, The Lancet Neurology, receiving free subscription; Editor-in-Chief, Multiple Sclerosis Journal, honorarium from SAGE Publications; support for travel as Chair, Scientific Advisory Committee, International Progressive MS Alliance, and member, National MS 
Society (USA), Research Programs Advisory Committee. Received honoraria and support for travel for lecturing from EXCEMED and Almirall. A.J.T. acknowledges also support from the UCL/UCLH NIHR Biomedical Research Centre.

Armin Curt reports grants from European Union's Horizon 2020 No 681094, grants from Swiss State Secretariat for Education (No 15.0255), grants from ERA-NET NEURON grant (SILENCE - no: 31NE30_173667), grants from ERA-NET NEURON grant (hMRIofSCI no: 32NE30_173678), grants from Swiss National Science Foundation (Pain control systems no. 320030_169250), during the conduct of the study. 


\section{References}

1 Ellingson BM, Salamon N, Holly LT. Imaging techniques in spinal cord injury. World Neurosurg 2014; 82: $1351-8$.

2 Kumar Y, Hayashi D. Role of magnetic resonance imaging in acute spinal trauma: a pictorial review. BMC Musculoskelet Disord 2016; 17: 310.

3 Shah LM, Ross JS. Imaging of Spine Trauma. Neurosurgery 2016; 79: 626-42.

4 Fehlings MG, Martin AR, Tetreault LA, et al. A Clinical Practice Guideline for the Management of Patients With Acute Spinal Cord Injury: Recommendations on the Role of Baseline Magnetic Resonance Imaging in Clinical Decision Making and Outcome Prediction. Glob Spine J 2017; 7: 221S 230S.

5 Freund P, Friston K, Thompson AJ, et al. Embodied neurology: an integrative framework for neurological disorders. Brain 2016; 139: 1855-61.

Huber E, David G, Thompson AJ, Weiskopf N, Mohammadi S, Freund P. Dorsal and ventral horn atrophy is associated with clinical outcome after spinal cord injury. Neurology 2018; 90: 10.1212/WNL.0000000000005361. Grabher P, Callaghan MF, Ashburner J, et al. Tracking sensory system atrophy and outcome prediction in spinal cord injury. Ann Neurol 2015; 78: 751-61.

8 Freund P, Weiskopf N, Ashburner J, et al. MRI investigation of the sensorimotor cortex and the corticospinal tract after acute spinal cord injury: a prospective longitudinal study. Lancet Neurol 2013; 12: $873-81$. Ziegler G, Grabher P, Thompson AJ, et al. Progressive neurodegeneration following spinal cord injury: Implications for clinical trials. Neurology 2018; 90: e1257-66.

10 Seif M, Curt A, Thompson AJ, Grabher P, Weiskopf N, Freund P. Quantitative MRI of rostral spinal cord and brain regions is predictive of functional recovery in acute Spinal cord injury. NeuroImage Clin 2018; published online Aug 19. DOI:10.1016/J.NICL.2018.08.026.

11 Ilvesmäki T, Koskinen E, Brander A, Luoto T, Öhman J, Eskola H. Spinal cord injury induces widespread chronic changes in cerebral white matter. Hum Brain Mapp 2017; 3647: 3637-47.

12 Koskinen E, Brander A, Hakulinen U, et al. Assessing the State of Chronic Spinal Cord Injury Using Diffusion Tensor Imaging. J Neurotrauma 2013; 30: 1587-95.

13 Koskinen EA, Hakulinen U, Brander AE, Luoto TM, Ylinen A, Öhman JE. Clinical correlates of cerebral diffusion tensor imaging findings in chronic traumatic spinal cord injury. Spinal Cord 2014; 52: 202-8.

Jirjis MB, Vedantam A, Budde MD, Kalinosky B, Kurpad SN, Schmit BD. Severity of spinal cord injury influences diffusion tensor imaging of the brain. J Magn Reson Imaging 2016; 43: 63-74. Cohen-Adad J. Microstructural imaging in the spinal cord and validation strategies. Neuroimage 2018; published online April 7. DOI:10.1016/j.neuroimage.2018.04.009. Cadotte DW, Akbar MA, Fehlings MG, Stroman PW, Cohen-Adad J. What Has Been Learned from Magnetic Resonance Imaging Examination of the Injured Human Spinal Cord: A Canadian Perspective. J Neurotrauma 2018; 35: 1942-57.

17 Trampel R, Bazin PL, Pine K, Weiskopf N. In-vivo magnetic resonance imaging (MRI) of laminae in the human cortex. Neuroimage. 2017; published online Sept 20.

DOI:10.1016/j.neuroimage.2017.09.037.

18 Lerch JP, van der Kouwe AJW, Raznahan A, et al. Studying neuroanatomy using MRI. Nat Neurosci 2017; 20: 314-26.

19 Hou J-M, Sun T-S, Xiang Z-M, et al. Alterations of resting-state regional and network-level neural function after acute spinal cord injury. Neuroscience 2014; 277: 446-54.

20 Zhu L, Wu G, Zhou X, Li J, Wen Z, Lin F. Altered Spontaneous Brain Activity in Patients with Acute Spinal Cord Injury Revealed by Resting-State Functional MRI. PLoS One 2015; 10: e0118816. Hawasli AH, Rutlin J, Roland JL, et al. Spinal Cord Injury Disrupts Resting-State Networks in the Human Brain. J Neurotrauma 2018; 35: 864-73.

22 Stroman PW, Khan HS, Bosma RL, et al. Changes in Pain Processing in the Spinal Cord and Brainstem after Spinal Cord Injury Characterized by Functional Magnetic Resonance Imaging. J Neurotrauma 2016; 33: 1450-60.

23 Zhong X-P, Chen Y-X, Li Z-Y, Shen Z-W, Kong K-M, Wu R-H. Cervical spinal functional magnetic resonance imaging of the spinal cord injured patient during electrical stimulation. Eur Spine J 2017; 26: 71-7. 
Barry RL, Conrad BN, Smith SA, Gore JC. A practical protocol for measurements of spinal cord functional connectivity. Sci Rep 2018; 8: 16512.

26 Kirshblum SC, Waring W, Biering-Sorensen F, et al. Reference for the 2011 revision of the International Standards for Neurological Classification of Spinal Cord Injury. J Spinal Cord Med 2011; 34: 547-54.

Talbott JF, Whetstone WD, Readdy WJ, et al. The Brain and Spinal Injury Center score: a novel, simple, and reproducible method for assessing the severity of acute cervical spinal cord injury with axial T2-weighted MRI findings. J Neurosurg Spine 2015; 23: 1-10. Aarabi B, Sansur CA, Ibrahimi DM, et al. Intramedullary Lesion Length on Postoperative Magnetic Resonance Imaging is a Strong Predictor of ASIA Impairment Scale Grade Conversion Following Decompressive Surgery in Cervical Spinal Cord Injury. Neurosurgery 2017; 80: 610-20. Jungmann PM, Agten CA, Pfirrmann CW, Sutter R. Advances in MRI around metal. J Magn Reson Imaging 2017; 46: 972-91.

30 Sundseth J, Jacobsen EA, Kolstad F, Nygaard OP, Zwart JA, Hol PK. Magnetic resonance imaging evaluation after implantation of a titanium cervical disc prosthesis: a comparison of 1.5 and 3 Tesla magnet strength. Eur Spine J 2013; 22: 2296-302.

31 Lee YH, Lim D, Kim E, Kim S, Song H-T, Suh J-S. Feasibility of fat-saturated T2-weighted magnetic resonance imaging with slice encoding for metal artifact correction (SEMAC) at 3T. Magn Reson Imaging 2014; 32: 1001-5. encoding for metal artefact correction with dual-source parallel radiofrequency excitation MRI at $3.0 \mathrm{~T}$. Br J Radiol 2013; 86: 20120524.

33 Worters PW, Sung K, Stevens KJ, Koch KM, Hargreaves BA. Compressed-sensing multispectral imaging of the postoperative spine. J Magn Reson Imaging 2013; 37: 243-8. Dalkilic T, Fallah N, Noonan VK, et al. Predicting Injury Severity and Neurological Recovery after Acute Cervical Spinal Cord Injury: A Comparison of Cerebrospinal Fluid and Magnetic Resonance Imaging Biomarkers. J Neurotrauma 2018; 35: 435-45. of Cervical Spinal Cord Injury Secondary to Trauma. World Neurosurg 2017; 101: 623-32. Matsushita A, Maeda T, Mori E, et al. Subacute T1-low intensity area reflects neurological prognosis for patients with cervical spinal cord injury without major bone injury. Spinal Cord 2016; 54: 24-8. Le E, Aarabi B, Hersh DS, et al. Predictors of intramedullary lesion expansion rate on MR images of patients with subaxial spinal cord injury. J Neurosurg Spine 2015; 22: 611-21.

38 Farhadi HF, Kukreja S, Minnema A, et al. Impact of Admission Imaging Findings on Neurological Outcomes in Acute Cervical Traumatic Spinal Cord Injury. J Neurotrauma 2018; 35: 1398-406. Aarabi B, Olexa J, Chryssikos T, et al. Extent of Spinal Cord Decompression in Motor Complete (American Spinal Injury Association Impairment Scale Grades A and B) Traumatic Spinal Cord Injury Patients: Post-Operative Magnetic Resonance Imaging Analysis of Standard Operative Approaches. $J$ Neurotrauma 2018; : neu.2018.5834. Haefeli J, Mabray MC, Whetstone WD, et al. Multivariate Analysis of MRI Biomarkers for Predicting Neurologic Impairment in Cervical Spinal Cord Injury. Am J Neuroradiol 2017; 38: 648-55. Mabray MC, Talbott JF, Whetstone WD, et al. Multidimensional Analysis of Magnetic Resonance Imaging Predicts Early Impairment in Thoracic and Thoracolumbar Spinal Cord Injury. J Neurotrauma 2016; 33: 954-62. Huber E, Lachappelle P, Sutter R, Curt A, Freund P. Are midsagittal tissue bridges predictive of outcome after cervical spinal cord injury? Ann Neurol 2017; 81: 740-8.

43 Dhall SS, Haefeli J, Talbott JF, et al. Motor Evoked Potentials Correlate With Magnetic Resonance Imaging and Early Recovery After Acute Spinal Cord Injury. Neurosurgery 2018; 82: 870-6. O'Dell DR, Weber KA, Berliner JC, et al. Midsagittal tissue bridges are associated with walking ability in incomplete spinal cord injury: A magnetic resonance imaging case series. J Spinal Cord Med 2018; : $1-4$.

Farhadi HF, Minnema AJ, Talbott JF, Aarabi B. Letter to the Editor: What Has Been Learned from Magnetic Resonance Imaging Examination of the Injured Human Spinal Cord: A Canadian Perspective. J Neurotrauma 2018; : neu.2018.6135.

46 Hou J, Xiang Z, Yan R, et al. Motor recovery at 6 months after admission is related to structural and functional reorganization of the spine and brain in patients with spinal cord injury. Hum Brain Mapp 2016; 37: 2195-209. Prados F, Ashburner J, Blaiotta C, et al. Spinal cord grey matter segmentation challenge. Neuroimage 2017; 152: 312-29. 
enlargement after cervical injury. Neurology 2019; : 10.1212/WNL.0000000000007137.

cord injury. Sci Rep 2016; 6: 18534

Chen Q, Zheng W, Chen X, et al. Brain Gray Matter Atrophy after Spinal Cord Injury: A Voxel-Based Morphometry Study. Front Hum Neurosci 2017; 11: 211.

Grabher P, Blaiotta C, Ashburner J, Freund P. Relationship between brainstem neurodegeneration and clinical impairment in traumatic spinal cord injury. NeuroImage Clin 2017; 15: 494-501.

Mole TB, MacIver K, Sluming V, Ridgway GR, Nurmikko TJ. Specific brain morphometric changes in spinal cord injury with and without neuropathic pain. Elsevier 2014; 5: 28-35.

Seif M, Ziegler G, Freund P. Progressive ventricles enlargement and CSF volume increases as a marker of neurodegeneration in SCI patients : A longitudinal MRI study. In: Ismrm. 2017: 2381.

Villiger M, Grabher P, Hepp-Reymond M-C, et al. Relationship between structural brainstem and brain plasticity and lower-limb training in spinal cord injury: a longitudinal pilot study. Front Hum Neurosci 2015; 9: 254.

Jutzeler CR, Freund P, Huber E, Curt A, Kramer JLK. Neuropathic Pain and Functional Reorganization in the Primary Sensorimotor Cortex after Spinal Cord Injury. J Pain 2015; 16. DOI:10.1016/j.jpain.2015.08.008.

Stroman PW, Wheeler-Kingshott C, Bacon M, et al. The current state-of-the-art of spinal cord imaging: Methods. Neuroimage 2014; 84: 1070-81.

Edwards LJ, Kirilina E, Mohammadi S, Weiskopf N. Microstructural imaging of human neocortex in vivo. Neuroimage 2018; 182: 184-206.

Brennan FH, Cowin GJ, Kurniawan ND, Ruitenberg MJ. Longitudinal assessment of white matter pathology in the injured mouse spinal cord through ultra-high field (16.4T) in vivo diffusion tensor imaging. Neuroimage 2013; 82: 574-85. spinal cord: insights from animal and human studies. Neurosurgery 2014; 74: 1-8; discussion 8; quiz 8. Novikov DS, Kiselev VG, Jespersen SN. On modeling. Magn Reson Med 2018; 79: 3172-93.

61 Lutti A, Bullmore ET, Weiskopf N, et al. Quantitative multi-parameter mapping of R1, PD*, MT, and R2* at 3T: a multi-center validation. Front Neurosci 2013; 7: 95. Wang F, Li K, Mishra A, Gochberg D, Min Chen L, Gore JC. Longitudinal assessment of spinal cord injuries in nonhuman primates with quantitative magnetization transfer. Magn Reson Med 2016; 75: 1685-96. Langkammer C, Krebs N, Goessler W, et al. Quantitative MR Imaging of Brain Iron: A Postmortem Validation Study 1. Radiology 2010; 257: 455-62.

64 Vedantam A, Eckardt G, Wang MC, Schmit BD, Kurpad SN. Clinical Correlates of High Cervical Fractional Anisotropy in Acute Cervical Spinal Cord Injury. World Neurosurg 2015; 83: 824-8. De Leener B, Fonov VS, Collins DL, Callot V, Stikov N, Cohen-Adad J. PAM50: Unbiased multimodal template of the brainstem and spinal cord aligned with the ICBM152 space. Neuroimage 2018; 165: 170-9.

David G, Freund P, Mohammadi S. The efficiency of retrospective artifact correction methods in improving the statistical power of between-group differences in spinal cord DTI. Neuroimage 2017; 158: 296-307.

67 Mohammadi S, Freund P, Feiweier T, Curt A, Weiskopf N. The impact of post-processing on spinal cord diffusion tensor imaging. Neuroimage 2013; 70: 377-85.

68 De Leener B, Lévy S, Dupont SM, et al. SCT: Spinal Cord Toolbox, an open-source software for processing spinal cord MRI data. Neuroimage 2017; 145: 24-43. Callaghan MF, Freund P, Draganski B, et al. Widespread age-related differences in the human brain microstructure revealed by quantitative magnetic resonance imaging. Neurobiol Aging 2014; 35: $1862-$ 72.

70 Sauerbeck A, Schonberg DL, Laws JL, McTigue DM. Systemic iron chelation results in limited functional and histological recovery after traumatic spinal cord injury in rats. Exp Neurol 2013; 248: 53-61.

71 Ahuja CS, Wilson JR, Nori S, et al. Traumatic spinal cord injury. Nat Rev Dis Prim 2017; 3: 17018.

72 Shanmuganathan K, Zhuo J, Chen HH, et al. Diffusion Tensor Imaging Parameter Obtained during Acute Blunt Cervical Spinal Cord Injury in Predicting Long-Term Outcome. J Neurotrauma 2017; 34: 2964-71.

73 Hur JW, Cho T-H, Park D-H, Lee J-B, Park J-Y, Chung Y-G. Intrathecal transplantation of autologous adipose-derived mesenchymal stem cells for treating spinal cord injury: A human trial. J Spinal Cord Med 2016; 39: 655-64. 
autologous transplantation of bone marrow mesenchymal stem cells in subjects with chronic spinal cord injury. Stem Cell Res Ther 2014; 5: 126.

Anderson KD, Guest JD, Dietrich WD, et al. Safety of Autologous Human Schwann Cell

Transplantation in Subacute Thoracic Spinal Cord Injury. J Neurotrauma 2017; 34: neu.2016.4895.

Zhou X, Sakaie KE, Debbins JP, et al. Quantitative quality assurance in a multicenter HARDI clinical trial at 3T. Magn Reson Imaging 2017; 35: 81-90.

77 Martin AR, Aleksanderek I, Cohen-Adad J, et al. Translating state-of-the-art spinal cord MRI techniques to clinical use: A systematic review of clinical studies utilizing DTI, MT, MWF, MRS, and fMRI. NeuroImage Clin 2016; 10: 192-238. 2013; 12: 843-4.

Zhou X, Sakaie KE, Debbins JP, Narayanan S, Fox RJ, Lowe MJ. Scan-rescan repeatability and crossscanner comparability of DTI metrics in healthy subjects in the SPRINT-MS multicenter trial. Magn Reson Imaging 2018; 53: 105-11.

80 Samson RS, Lévy S, Schneider T, et al. ZOOM or Non-ZOOM? Assessing spinal cord diffusion tensor imaging protocols for multi-centre studies. PLoS One 2016; 11: e0155557.

81 Seif M, Leutritz T, Samson RS, et al. A multi-center study on fast full-brain quantitative multiparameter mapping of R1, MT, and R2*: scan-rescan repeatability and inter-site reproducibility. In: ISMRM. 2018: 1119. Kucher K, Johns D, Maier D, et al. First-in-Man Intrathecal Application of Neurite Growth-Promoting Anti-Nogo-A Antibodies in Acute Spinal Cord Injury. Neurorehabil Neural Repair 2018; 32: 578-89. Eippert F, Kong Y, Winkler AM, et al. Investigating resting-state functional connectivity in the cervical spinal cord at 3T. Neuroimage 2017; 147: 589-601. Solstrand Dahlberg L, Becerra L, Borsook D, Linnman C. Brain changes after spinal cord injury, a quantitative meta-analysis and review. Neurosci Biobehav Rev 2018; 90: 272-93. Jutzeler CR, Curt A, Kramer JLK. Relationship between chronic pain and brain reorganization after deafferentation: A systematic review of functional MRI findings. NeuroImage Clin 2015; 9: 599-606. Wrigley PJ, Siddall PJ, Gustin SM. New evidence for preserved somatosensory pathways in complete spinal cord injury: A fMRI study. Hum Brain Mapp 2018; 39: 588-98. 8: 173 . Zhao W, Cohen-Adad J, Polimeni JR, et al. Nineteen-channel receive array and four-channel transmit array coil for cervical spinal cord imaging at 7T. Magn Reson Med 2014; 72: 291-300.

89 Finsterbusch J, Sprenger C, Büchel C. Combined T2*-weighted measurements of the human brain and cervical spinal cord with a dynamic shim update. Neuroimage 2013; 79: 153-61.

90 Min Y-S, Park JW, Jin SU, et al. Alteration of Resting-State Brain Sensorimotor Connectivity following Spinal Cord Injury: A Resting-State Functional Magnetic Resonance Imaging Study. J Neurotrauma 2015; 32: 1422-7.

91 Oni-Orisan A, Kaushal M, Li W, et al. Alterations in Cortical Sensorimotor Connectivity following Complete Cervical Spinal Cord Injury: A Prospective Resting-State fMRI Study. PLoS One 2016; 11: e0150351.

92 Kaushal M, Oni-Orisan A, Chen G, et al. Evaluation of whole-brain resting-state functional connectivity in spinal cord injury - a large-scale network analysis using network based statistic. J Neurotrauma 2016; : neu.2016.4649.

93 Barry RL, Smith SA, Dula AN, Gore JC. Resting state functional connectivity in the human spinal cord. Elife 2014; 2014: 1-15.

94 Sprenger C, Finsterbusch J, Buchel C. Spinal Cord-Midbrain Functional Connectivity Is Related to Perceived Pain Intensity: A Combined Spino-Cortical fMRI Study. J Neurosci 2015; 35: 4248-57. Duval T, Lévy S, Stikov N, et al. g-Ratio weighted imaging of the human spinal cord in vivo. Neuroimage 2017; 145: 11-23.

96 Mohammadi S, Carey D, Dick F, et al. Whole-brain in-vivo measurements of the axonal G-ratio in a group of 37 healthy volunteers. Front Neurosci 2015; 9: 1-13.

97 Morawski M, Kirilina E, Scherf N, et al. Developing 3D microscopy with CLARITY on human brain tissue: Towards a tool for informing and validating MRI-based histology. Neuroimage 2018; 182: 41728. white matter: an electron-microscopic study on three human brains and a macaque. Biol Cybern 2014; 108: 541-57. 


\section{Figure captions}

\section{Figure 1: The BASIC score}

The BASIC score of SCIs comprises a 5-point ordinal MRI score for classifying acute SCIs on the basis of conventional axial T2-weighted scans. The BASIC score stratifies SCI according to the extent of transverse T2-weighted signal abnormality during the acute phase of the injury. Cartoon schematics (A), representative axial T2-weighted MRI scans (B), 3Dcolor surface plots based on the axial T2-weighted MRI (C), and brief definitions (D) for each of the 5 BASIC scores (ranging from 0 to 4). In the representative MRI scans (B), the external contour of the spinal cord is outlined in yellow for better delineation. (Reprinted from Talbott et al., $2015^{27}$ )

\section{Figure 2: Extent of lesion and tissue bridges}

Structural magnetic resonance imaging (MRI) indices assessed at the lesion site. A: Postoperative T2-weighted MRI of the cervical cord from a 17-year-old male patient with traumatic spinal cord injury (male, ASIA Impairment Scale (AIS) grade of A (complete)). Postoperative MRI at 32.5 hours after operation indicates an intramedullary lesion length (IMLL) of $102 \mathrm{~mm}$ (long bracket) with bleeding (short bracket) and myelomalacia (dotted lines) at the injury epicentre (Reprinted from Aarabi Bizhan et al., $2017^{28}$ with permission from American Association for the Advancement of Science). B: Demonstration of the lesion segmentation using mid-sagittal T2-weighted MRI within the cervical cord of a 51 year-old incomplete SCI patient (male, tetraplegic, AIS grade of C and with scan finding of central T2-weighted hyperintensive cord defect at the C6/C7 level). C: Demonstration of the lesion segmentation using mid-sagittal T2-weighted MRI within the thoracic cord of an 80-year-old incomplete SCI patient (female, paraplegic, AIS grade of C and with scan finding of subdural haemorrhage on T4 level). D: Schematic drawing of the lesion on the cord for analysing the lesion parameters, $\mathrm{DB}=$ dorsal midsagittal tissue bridges; $\mathrm{LA}=$ lesion area; $\mathrm{LL}=$ lesion length; $\mathrm{LW}=$ lesion width; $\mathrm{VB}=$ ventral midsagittal tissue bridges.

Figure 3: The lesion evolution over time Please provide figure title

Overview of the lesion evolution with persisting midsagittal tissue bridges over 1-year postinjury. Longitudinal T2-weighted sagittal MRI scans showing the evolution of the cervical lesion epicenter from a 27-year-old complete SCI patient (male, tetraplegic, AIS grade A) in 
acute (one day post -SCI), subacute (1 month post-SCI) and chronic (12 months post-SCI) phase after injury.

Figure 4: Schematic representation of connections between qMRI methods and the neocortical microstructural features

Relationships between different quantitative MRI readouts and the microstructural features reported previously in post mortem brain tissue $\mathrm{e}^{97,98}$ to which they have been linked. A coloured line between a quantitative MRI readout and a microstructural feature implies that this readout has been empirical linked to this feature. The relationships between MR contrast and microstructural features makes microstructural mapping through the combination of complementary quantitative MR images possible. MT = magnetisation transfer saturation; $\mathrm{PD}=$ proton density, $\mathrm{R} 1=$ longitudinal relaxation rate, $\mathrm{R} 2 *$ = effective transverse relaxation rate, $\mathrm{QSM}=$ quantitative susceptibility mapping, $\mathrm{R} 2=$ transverse relaxation rate, $\mathrm{dMRI}=$ diffusion weighted MRI. (Reprinted from Edwards et al. $2018{ }^{57}$ ) under the terms of the CCBY 4.0 license (https://creativecommons.org/licenses/by/4.0/)).

\section{Figure 5: Schematic representation of primary and secondary degenerative processes} occurring remotely, above and below the injury site

Primary damage at the focal epicenter of the lesion occurring within hours after injury $\mathbf{A}$, and secondary systematic degenerative processes occur remotely $\mathbf{B}$, above, below and at the primary injury site. Sensory and motor tracts affected by the injury undergo anterograde and/or retrograde (depending on the location) axonal degeneration and accompanying demyelination. ${ }^{6,12,64}$ Lesion site shows the macrostructural evidence of primary intramedullary damage (e.g. oedema $\&$ haemorrhage) and secondary changes such as posttraumatic cyst cavities and spared tissue bridges ${ }^{42,44}$ In the lumbar cord, the lower motor neurons located in the ventral horn may undergo trans-synaptic degeneration due to the loss of input from the injured corticospinal tracts. ${ }^{48}$ Similarly, second-order sensory neurons of the spinothalamic and dorsal column medial lemniscus systems can also be affected by transsynaptic degeneration. At the brain level, atrophic changes are located within the brainstem, ${ }^{51}$ cranial corticospinal tracts, primary motor cortices, insula, anterior cingulate cortex, and thalamus. ${ }^{7,8,14}$ Some of these areas (e.g. cortical and subcortical regions) present also with 
changes in myelin and iron content which is suggestive of demyelination and iron accumulation. $^{9}$

DTI: diffusion tensor imaging, MPM: multi-parameter mapping, T1w and T2*w MRI: T1 weighted and $\mathrm{T} 2 *$ weighted magnetic resonance imaging, Add abbreviations for T1w MRI, etc

Table 1: Completed clinical trials in spinal cord injury (SCI) using MRI as an outcome measure within the last 5 years

\begin{tabular}{|c|c|c|c|c|c|c|c|}
\hline \multirow{2}{*}{$\begin{array}{c}\text { Clinical } \\
\text { trials.gov } \\
\text { identifier }\end{array}$} & \multirow[t]{2}{*}{ Study Title } & \multirow[t]{2}{*}{ Intervention } & \multirow{2}{*}{$\begin{array}{c}\text { Enrollment (number } \\
\text { of } \\
\text { Participants)/Condit } \\
\text { ion }\end{array}$} & \multicolumn{2}{|c|}{ MRI as outcome measures } & \multirow{2}{*}{ MRI techniques } & \multirow{2}{*}{$\begin{array}{c}\text { Status } \\
\text { Primary Date }\end{array}$} \\
\hline & & & & $\begin{array}{l}\text { Primary } \\
\text { outcome }\end{array}$ & $\begin{array}{c}\text { Secondary } \\
\text { outcome }\end{array}$ & & \\
\hline NCT01325103 & $\begin{array}{l}\text { Evaluation of } \\
\text { Autologous } \\
\text { Mesenchymal } \\
\text { Stem Cell } \\
\text { Transplantation in } \\
\text { Chronic Spinal } \\
\text { Cord Injury: } \\
\end{array}$ & $\begin{array}{l}\text { Autologous } \\
\text { Mesenchymal Stem } \\
\text { Cell }\end{array}$ & $\begin{array}{l}14 \text { Participants } \\
\text { chronic spinal cord } \\
\text { injury }\end{array}$ & & $\begin{array}{l}\nabla \text { Lesion } \\
\text { volume }\end{array}$ & $\begin{array}{l}\text { Conventional MRI } \\
\text { scan (T1/T2 } \\
\text { weighted MRI) }\end{array}$ & $\begin{array}{l}\text { Completed } \\
\text { December } \\
2012 \\
\square \text { Published } \\
\text { results }{ }^{74}\end{array}$ \\
\hline NCT01624779 & $\begin{array}{l}\text { Intrathecal } \\
\text { Transplantation of } \\
\text { Autologous } \\
\text { Adipose Tissue } \\
\text { Derived MSC in } \\
\text { the Patients With } \\
\text { Spinal Cord } \\
\text { Injury }\end{array}$ & $\begin{array}{l}\text { Autologous adipose } \\
\text { tissue derived } \\
\text { mesenchymal stem } \\
\text { cells }\end{array}$ & $\begin{array}{l}15 \text { Participants } \\
\text { chronic spinal cord } \\
\text { injury }\end{array}$ & $\begin{array}{l}\text { Qualitative } \\
\text { lesion } \\
\text { assessment }\end{array}$ & & $\begin{array}{l}\text { Conventional MRI } \\
\text { scan (T1/T2 } \\
\text { weighted axial and } \\
\text { sagittal MRI) }\end{array}$ & $\begin{array}{l}\text { Completed } \\
\text { May } 2014 \\
\square \text { Published } \\
\text { results } 73\end{array}$ \\
\hline NCT01739023 & $\begin{array}{l}\text { Safety of } \\
\text { Autologous } \\
\text { Human Schwann } \\
\text { Cells (ahSC) in } \\
\text { Subjects with } \\
\text { Subacute SCI }\end{array}$ & $\begin{array}{l}\text { Autologous Human } \\
\text { Schwann Cell }\end{array}$ & $\begin{array}{l}9 \text { Participants } \\
\text { Subacute SCI ( } 30 \\
\text { day-post injury) }\end{array}$ & & $\begin{array}{l}\nabla \text { Lesion } \\
\text { volume }\end{array}$ & $\begin{array}{l}\text { Conventional MRI } \\
\text { scan (T1/T2 } \\
\text { weighted) MRI on } \\
\text { the lesion area }\end{array}$ & $\begin{array}{l}\text { Completed } \\
\text { August } 2016 \\
\square \text { Published } \\
\text { results }{ }^{75}\end{array}$ \\
\hline
\end{tabular}

Table2: Different MRI techniques applied in SCI and their corresponding outcome measures

\begin{tabular}{|c|c|}
\hline MRI techniques & Outcome measures \\
\hline $\begin{array}{l}\text { Sagittal and axial conventional T2-weigthed } \\
\text { MRI of the spinal cord at the injury level }\end{array}$ & $\begin{array}{l}\text { - } \quad \text { extent of the intramedullary injury \& lesion length }{ }^{27,28,38} \\
\text { - } \\
\text { - } \\
\text { - } \\
\text { oedemoma }^{36} \\
\text { spinal cord compression }^{99}\end{array}$ \\
\hline $\begin{array}{l}\text { Sagittal conventional T2-weigthed MRI of the } \\
\text { spinal cord at the injury level }\end{array}$ & - $\quad$ preserved midsagittal tissue bridges ${ }^{42,44}$ \\
\hline $\begin{array}{l}\text { T1-weighted MRI (above the injury level, } \\
\text { cervical cord and brain) }\end{array}$ & - $\quad$ cervical cord and brain atrophy \\
\hline $\begin{array}{l}\text { T2*-weighted MRI of the cervical and lumbar } \\
\text { cord (remote from the injury site) }\end{array}$ & - $\quad$ grey and white matter atrophy of the spinal cord ${ }^{6,48}$ \\
\hline $\begin{array}{l}\text { Diffusion tensor imaging (DTI) of the cord } \\
\text { and brain } \\
\text { (remote from the injury site) }\end{array}$ & - $\quad$ axonal degeneration \& demyelination ${ }^{6,11,12,14,64}$ \\
\hline
\end{tabular}




\begin{tabular}{|l|c|}
\hline $\begin{array}{l}\text { Multi parameter mapping (MPM) of the } \\
\text { cervical cord and brain (above the injury } \\
\text { level) }\end{array}$ & $\bullet \quad$ demyelination \& iron deposition $^{7-10}$ \\
\hline $\begin{array}{l}\text { Functional MRI (fMRI) of brain and spinal } \\
\text { cord (remote from injury level) }\end{array}$ & $\bullet \quad{\text { functional networks \& plasticity in brain }{ }^{19-21} \& \text { spinal cord }^{22-25}}$
\end{tabular}

\section{Panel}

\section{Clinical and electrophysiological assessments}

To date, the symptoms and signs of myelopathy (i.e., the degree of sensorimotor deficit and the emergence of neuropathic pain) are assessed clinically and by electrophysiological tests. The current gold standard in assessing clinical impairment is by means of the International Standards for the Neurological Classification of Spinal Cord Injury (ISNCSCI) protocol. ${ }^{1}$ This test is routinely performed at admission by a qualified clinician who tests key muscles for strength and all dermatomes for light-touch and pin-prick sensation. A score is then calculated which is used to classify patients' overall impairment into a scale with five ranked categories (ASIA impairment scale) - A to E. Category A is the most severely damaged spinal cord with no motor and sensory function below the level of injury whereas category $\mathrm{E}$ features no clinically relevant impairment. Although defining AIS categories is a fairly easy process, it does not capture the entire extent of primary and secondary injury mechanisms. Consequently, this leads to considerable heterogeneity within an AIS category, which limits its applicability as a surrogate endpoint in clinical trials. Thus, large clinical trials are needed to distinguish a treatment effect from natural history. To address this drawback, dedicated prediction models (i.e., unbiased recursive partitioning) have recently been established. ${ }^{2-4}$ These models aim to reduce the heterogeneity within SCI cohorts thereby improving patient stratification.

Recently, more refined measures have also been developed. For upper limb function, manual dexterity is assessed by the Graded Redefined Assessment of Strength Sensibility and Prehension (GRASSP) score ${ }^{5}$, functional independence by the functional independence 
(SCIM) score ${ }^{6}$, and neuropathic pain intensity is commonly assessed with pain questionnaires. ${ }^{7}$ Neurophysiological recordings such as motor and sensory evoked potentials ${ }^{8}$, as well as contact heat evoked potentials ${ }^{9}$, can provide additional information about the integrity of impulse conductance of motor and distinct sensory pathways. ${ }^{10}$ However, these examinations report on impaired function related to focal injury; they do not reflect remote neurodegeneration and functional reorganizational processes that occur with distinct (delayed) temporal profiles. ${ }^{11}$ However, all these examinations fail to differentiate or elucidate the mechanisms responsible for recovery processes in-vivo.

1 Kirshblum S, Waring W. Updates for the International Standards for Neurological Classification of Spinal Cord Injury. Phys Med Rehabil Clin N Am 2014; 25: 505-17, vii.

2 Tanadini LG, Hothorn T, Jones LAT, et al. Toward Inclusive Trial Protocols in Heterogeneous Neurological Disorders: Prediction-Based Stratification of Participants With Incomplete Cervical Spinal Cord Injury. Neurorehabil Neural Repair 2015; 29: 867-77.

3 Tanadini LG, Steeves JD, Hothorn T, et al. Identifying Homogeneous Subgroups in Neurological Disorders: Unbiased Recursive Partitioning in Cervical Complete Spinal Cord Injury. Neurorehabil Neural Repair 2014; 28: 507-15.

4 Velstra I-M, Bolliger M, Tanadini LG, et al. Prediction and Stratification of Upper Limb Function and Self-Care in Acute Cervical Spinal Cord Injury With the Graded Redefined Assessment of Strength, Sensibility, and Prehension (GRASSP). Neurorehabil Neural Repair 2014; 28: 632-42.

5 Velstra I-M, Curt A, Frotzler A, et al. Changes in Strength, Sensation, and Prehension in Acute Cervical Spinal Cord Injury. Neurorehabil Neural Repair 2015; 29: 755-66.

6 Itzkovich M, Shefler H, Front L, et al. SCIM III (Spinal Cord Independence Measure version III): reliability of assessment by interview and comparison with assessment by observation. Spinal Cord 2018; 56: 46-51.

7 Hatch MN, Cushing TR, Carlson GD, Chang EY. Neuropathic pain and SCI: Identification and treatment strategies in the 21 st century. J Neurol Sci 2018; 384: 7583.

8 Petersen JA, Spiess M, Curt A, et al. Upper Limb Recovery in Spinal Cord Injury: Involvement of Central and Peripheral Motor Pathways. Neurorehabil Neural Repair 2017; 31: 432-41.

9 Jutzeler CR, Ulrich A, Huber B, Rosner J, Kramer JLK, Curt A. Improved Diagnosis of Cervical Spondylotic Myelopathy with Contact Heat Evoked Potentials. $J$ Neurotrauma 2017; 34: 2045-53.

10 Hupp M, Pavese C, Bachmann LM, Koller R, Schubert M. Electrophysiological Multimodal Assessments Improve Outcome Prediction in Traumatic Cervical Spinal Cord Injury. J Neurotrauma 2018; : neu.2017.5576.

11 Freund P, Friston K, Thompson AJAJ, et al. Embodied neurology: an integrative 
framework for neurological disorders. Brain 2016; 139: 1855-61. 


\section{MRI in traumatic spinal cord injury: progress from a-clinical assessment tool to-z neuroimaging biomarkers}

Patrick Freund, $\mathrm{MD}, \mathrm{PhD}-\mathrm{PhD}^{1,2,3,4}$, Maryam Seif, $\mathrm{PhD} \mathrm{PhD}^{1,3}$, Nikolaus Weiskopf, $\mathrm{PhD}-\mathrm{PhD}^{3,4}$, Karl Friston, $\mathrm{MD}-\mathrm{MD}^{4}$, Michael G. Fehlings, MD-MD, Alan Thompson, MD-MD ${ }^{2}$ and Armin Curt, MD-MD,

1 Spinal Cord Injury Center Balgrist, University Hospital Zurich, University of Zurich, Zurich, Switzerland

${ }^{2}$ Department of Brain Repair \& Rehabilitation, UCL Institute of Neurology, UCL, London, UK

3 Department of Neurophysics, Max Planck Institute for Human Cognitive and Brain Sciences, Leipzig, Germany

${ }^{4}$ Wellcome Centre for Human Neuroimaging, UCL Institute of Neurology, UCL, London, UK

${ }^{5}$ University of Toronto Spine Program and Toronto Western Hospital, Toronto, Ontario, Canada

\section{Corresponding author:}

DrProf. Patrick Freund, Spinal Cord Injury Center, Balgrist University Hospital, Forchstrasse 340 8008 Zurich, Switzerland, E-mail: Patrick.Freund@ Balgrist.ch, Phone: +41 443861111
Formatted: German (Switzerland)

Formatted: German (Switzerland)

Formatted: German (Switzerland)

Formatted: Font: Bold, German

(Switzerland)

Formatted: Font: Bold, German (Switzerland)

Formatted: German (Switzerland)

Formatted: German (Switzerland)

Comment [WM(1]: Please add one degree qualification per author

Comment [MOU2]: Now changed

Formatted: German (Switzerland)

Formatted: German (Switzerland)

Formatted: German (Switzerland)

Formatted: German (Switzerland)

Formatted: German (Switzerland)

Formatted: German (Switzerland) 


\section{Abstract}

Traumatic spinal cord injury (SCI) occurs when an external physical impact acutely damages the spinal cord and leads to permanent neurologic dysfunction, personal disability and social burden. Conventional MRI plays a crucial eritical-role in the diagnostic workup of SCI patients and can guide clinical treatment-as it reveals extrinsic compression of the cord and disruption of the discoligamentous complexesseoligamentous spinenal elements.

Additionally, ift atse-can reveal macrostructural evidence of primary intramedullary damage such as haemorrhage, oedema, post-traumatic cystic cavities and spared-tissue bridges. Quantitative MRI (qMRI), such as magnetisation transfer, MR relaxation mapping and diffusion imaging, enables the tracking of secondary changes across the neuroaxisneuraxis at Comment [MS5]: Response to R2.4 the microstructural level. Both, conventional MRI and qMRI metrics, obtained early after SCI, are predictive of outcome. Thus, neuroimaging biomarkers may serve as surrogate endpoints for more efficient interventional-trials targeting the-acute and chronic stages-of injury $\underline{S C I}$. The adoption of neuroimaging biomarkers in elinical-SCI centres willenable the development of more efficient trials and-may eventually lead to individualized patient care approaches. 


\section{Introduction}

Conventional MRI of the spinal cord is an essential component in the diagnostic investigation, surgical treatment, and rehabilitation of patients with spinal cord injury (SCI). ${ }^{1}$ Spinal MRI is the gold standard for the evaluation of any damage to the disco-ligamentous complex diseo ligamentary (i.e. spine instability and spinal canal encroachment) and neural structures (i.e. integrity of the spinal cord) induced by mechanical trauma. ${ }^{2}$ In clinical practice sagittal and axial T2-weighted MRI sequences are usually applied and can be complemented with a short-T1 inversion recovery (STIR) sequence. ${ }^{3}$ These conventional MRI sequences reveal the level of the damage and the extent of intra/extramedullary abnormalities (oedema and haemorrhage), the degree of spinal cord compression, extent of disk herniation, and ligamentous instability at the level of the injury. ${ }^{3}$ Coupled with the clinical examination, these imaging findings obtained within hours of the trauma, guide decision making and lead to a timely and appropriate decompression of the contused and compressed spinal cord. ${ }^{4}$

Despite their critical importance in clinical management, these conventional MRI sequences provide relatively less information about the evolving microstructural changes of the immediate and adjacent spinal cord segments and, subsequently, of the brain. There is a pressing need for a more in-depth understanding of both the complex processes of neural plasticity, at the microstructural level, and the complex functional interactions between spinal and supraspinal networks involved in SCI recovery. ${ }^{5}$ Such information can help us to understand the more in depthpathobiology as it-disease processes and enables tothe ean
Comment [WM(6]: It should be made clear here and, in the discussion, (something along these lines) that until recently, past clinical markers (AIS and AMS) were the only variables used as predictors of outcome following spinal cord injury. Now structural MRI imaging markers such as the Basic Score and intramedullary lesion length (IMLL) were considered as useful predictors of outcome. Based on your review is seems that the future belongs to better understanding qualitative MRI (qMRI: magnetization transfer, relaxation maps and diffusion characteristics) and using it as an indicator of outcome.

Comment [MOU7]: We have now amended the introduction

Comment [MS8]: Response to R2.2 
tracking of tissteneuronal changes at-integrity in the microstructural level across the disease processneuraxis. -[A: please make more explicit why there is a need] Recent $s$ Spinal imaging studies from a number of centres have employed advanced quantitative MRI (qMRI) techniques, such as magnetisation transfer, MR relaxation mapping, and diffusion imaging to improve detection and quantification of microstructural features of trauma-induced pathology both at and remotely from the site of injury. ${ }^{6-14}$ These qMRI protocols provide quantitative measures of spinal cord ${ }^{15,16}$ and brain integrity ${ }^{17}$ that reflect atrophy, demyelination, and iron deposition of tissue. They have been used to demonstrate widespread ${ }^{6-14}$ and progressive neurodegeneration $;^{7-9}$ the magnitude of which predicts clinical recovery. ${ }^{9,10}$ qQMRI therefore offers improved assessments of underlying neural integrity and can provide insights into the relationship between clinical recovery and neural plasticity, within the spinal cord and the brain. ${ }^{18}$ Additionally, task-based (fMRI) and resting state (rs-fMRI) functional MRI, although non-quantitative, can probe plasticity at the level of the brain, ${ }^{19-21}$ and more recently within the spinal cord. ${ }^{22-25}$ In clinical practice, sensorimotor impairments assessed by means of the International Standards for Neurological Classification of Spinal Cord Injury $\underline{(I S N C S C I}^{26}$ are commonly used as predictors of outcome following SCI. Now conventional $\underline{\text { MRI markers such as the Basic Score }}{ }^{27}$ and intramedullary lesion length (IMLL) ${ }^{28}$ wereare considered as useful predictors of outcome. However, the future belongsportends ato better understanding of SCItrauma-induced microstructural changes by means of qMRI (e.g. magnetization transfer, relaxation maps and diffusion characteristics) and potentially usinga potential use of qMRI as an indicator of outcome in clinical trials.

In this review, we evaluate recent findings from conventional MRI and discuss the insights they have provided concerning the primary pathological features of the injury epicentre. We then assess recent-developments in qMRI imaging studies that have shone new light on secondary pathological changes affecting the entire neuroaxisneuraxis. The relevance and 
implications of these advances for improving the ability to predict recovery are discussed, followed by an assessment of their application as biomarkers in trials of patients with acute $\_$

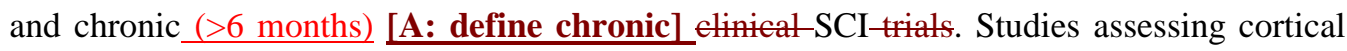
and spinal functional plasticity by means of fMRI and rs-fMRI are reviewed before providing recommendations concerning the application of MRI protocols in clinical and research settings. Finally, we suggest directions for further and-future research.

\section{Use of $\in$ Conventional MRIto reveal focal cord pathology}

\section{Immediate changes at the epicentre}

The majority of SCI patients undergo decompression surgery and receive spinal fixation devices (i.e. metallic implants) to manage spinal instability. The presence of metallic implants Such devices Italso causes significant MRI artifacts such as signal-loss, signal-pileup, geometric distortion, and failure of fat suppression, ${ }^{29}$ which worsen with increasing magnetic field strength. ${ }^{30}$ These image artefacts utility and reduce the quality of the qMRI metrics. [A: what are the implications of these artefacts as this might not be obvious for a non-expert]Current strategies for metal artefact suppression, that allow scan acquisitions in a clinically feasible duration, include the slice-encoding for metal artefact correction ${ }^{31}$ with dual-source parallel radiofrequency, ${ }^{32}$ as well as compressed-sensing multi-spectral imaging techniques. ${ }^{33}$ By taking advantage of such techniques, recent spinal cord imaging studies have investigated primary changes (i.e. macrostructural) immediately following the injury, focally at the injury site and based on hyperintensity signal intensity changes of sagittal and axial T2-weighted_and hypointensity signal changes of T1-weighted_-MRI scans. ${ }^{28,34-39}$ The most prominent features on sagittal

T2-weighted scans include haemorrhage, cytotoxic oedema, and spinal cord swelling. ${ }^{28,37}$ 
Serial quantification of sagittal T2-weighted hyperintensity-ies revealed that the intramedullary damage dynamically expands rostrally and caudally, with injury severity [A:

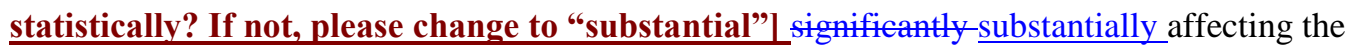
rate of expansion. ${ }^{27,28,37}$ Based on T2-weighted signal abnormalities, a 5-point ordinal MRI score referred to as the "BASIC score" has been proposed for MRI-based diagnostic and prognostic classification in patients with acute SCI. ${ }^{27}$ The BASIC score quantifies five distinct patterns of intramedullary T2-weighted signal abnormality in the axial plane at the injury epicentre of the spinal cord (Figure 1). These patterns range from no abnormalities to the most severe abnormalities consisting of mixed haemorrhage and oedema. The feasibility and prognostic validity of BASIC scores have been demonstrated both for patients with acute cervical $^{27,40}$ and thoracic ${ }^{41}$ SCI, where MRI had been performed within days after injury. Moreover, the intramedullary lesion size, measured on sagittal T2-weighted slices (Figure 2 A), is a good predictor of recovery as its size is influenced by injury severity ${ }^{34-38}$ and the outcome of surgical decompression. ${ }^{28}$

A caveat of quantifying intramedullary damage using conventional MRI scans is the nonspecificity of T2-weigthed signal changes to the underlying pathophysiology. T2-weighted signal changes may reflect various processes, including oedema, inflammation, and the development of myelomalacia, demyelination, or cyst cavitation. ${ }^{27}$ Moreover, interpretation should be dependent on the timing of MRI assessments as the evolution of oedema and haemorrhage changes considerably and is highly variable across subjects-patients. ${ }^{28}$ Finally, the quantification of changes in T2-weigthed MRI is usually performed manually by a user with 3 years of experience in processing conventional MRI [A: by whom?], as fully automated methods that can reliably distinguish artefact-induced signal changes at the epicentre of the -a traumatic lesion are currently lacking absent [A: scare or absent?]. Thus, 
the utility of the BASIC score, as well as the quantification of the intramedullary lesion length, needs to berequires further later time points would be ideal, for example, during rehabilitation where the T2-weighted signal abnormalities have evolved. ${ }^{42}$

\section{Evolution of changes at the epicentre}

A longitudinal study of thirteen SCI patients with cervical injury [A: how many patients?] employing conventional MRI has investigated the natural sequelae of macrostructural intramedullary changes at the focal injury site during the first year post-SCI ${ }^{42}$. T2-weigthed scans showed a transition from the acute oedema and haemorrhage ${ }^{43}$ to sub-acute intramedullary lesion expansion ${ }^{34,37}$ (Figure 3). After signs of oedema and haemorrhage slowly evolved, a post-traumatic cyst appeared in in the majorityall of [A: if possible, please add exact patient number and percentage] thirteen patients within the first month post$\mathrm{SCI}^{42}$ At this stage it is possible to detect small tissue bridges around the post-traumatic cyst that can be measured at the dorsal and ventral aspect of the cord, adjacent to the cyst on midsagittal T2-weighted scans (Figure 2 B-D) ${ }^{42,44}$ Crucially, the width and location of these tissue bridges predict tract-specific electrophysiological information flow ${ }^{42}$ and long-term functional recovery. ${ }^{42,44}$ Thus, the quantification of spared midsagittal tissue bridges on $\mathrm{T} 2$ weighted scans, at 1-month post-SCI, holds potential as an important prognostic tool.

The potential predicting ability of theMRI markers of -lesion epicentre-characteristics as well $\underline{\text { as tissue bridges }}$ bove outlined $[$ A: please specify $]$ evidence_-emphasises the importance of currently available and cost effective-[A: you haven't mentioned previously that they are cost-effective] conventional MRI protocols to be applied in the clinical particular, T2-based scans can detect dynamic intramedullary signal changes as well as preserved midsagittal tissue bridges. ${ }^{42}$ [A: please add supporting ref] Both can serve as an 
important diagnostic and prognostic tools, being sensitive to therapeutic interventions.

Conventional MRI protocols are also easily applied in longitudinal designs at any stage of SCI and thus could furnish neuroimaging biomarkers for clinical trials. ${ }^{42}$

\section{Conventional MRI: Tracking remote cord and brain atrophy \\ Progressive cord atrophy}

Despite these insights provided by manually quantifying the primary effects of the trauma at the epicentre of the injury, automated and unbiased quantification of trauma-induced changes at the level of the injury are still not feasible mostlylargely due to the artefacts induced by metal implants at the lesion site -[A: why?]. One strategy to measure structural changes free of metal artefacts and hence capable of being performed fully or semi-automatically $-y$, is to target the artefact free spinal cord above and below the level of injury. A prospective, longitudinal MRI investigation of XXfifteen traumatic SCI patients patientswith SCI, tracking changes to the cross-sectional cord area (measured in $\mathrm{mm}^{2}$ ) based on a T1-weigthed MPRAGE sequence at the cervical cord level (C2/C3), showed signs of remote_spinal-[A: ok? If yes, please add "spinal" throughout the manuscriptl_eord atrophyspinal cord atrophy within months-two months [A: could you be more precise - how many months?] of the SCI. ${ }^{8,46}$ Over time (one year post SCI) [A: could you be more precise - how many months?], atrophy continues to progress, ${ }^{7,8}$ at the level of the cervical cord, reaching $14 \%$ reduced cross-sectional cord area compared with healthy controls [A: meaning not entirely clear] in the chronic phase post-SCI. ${ }^{6,9}$ Interestingly, the rate of cord atrophyspinal cord atrophy only showed signs of deceleration two years later[A: how many years?]. ${ }^{9}$ In the chronic state of SCI, high resolution multi-echo gradient echo $72 *$ weighted-scans-, , that allow to segment the grey and white matter of the cord, ${ }^{47}$ showed that remote neurodegeneration occurs within the dorsal and ventral horns as well as white matter within 
the high cervical cord ${ }^{6}$ and lumbar enlargement. ${ }^{48}$ While dorsal horn atrophy at the cervical level was associated with sensory outcome_(e.g. ISNCSCI pin-prick scores) (eg, XX) ҐA: please add], ventral horn atrophy was associated with $\underline{\text { ISNCSCI motor eutcome-score. }}{ }^{6}$ It is still not clear whether the rate of atrophy is related to the lesion level and/or injury severity. ${ }^{7,10,46}$ However, the magnitude of remote eord atrophyspinal cord atrophy within the first six months post-SCI is predictive of functional recovery. ${ }^{9,10,46}$

\section{Progressive brain atrophy}

At the level of the brain, the conventional T1-weighted MPRAGE sequence that covers the brain and cervical cord, has provided insights into remote brain atrophy. Trauma-induced brain atrophy is particularly prominent across the cranial projections of the corticospinal tracts, primary motor cortex, insula, anterior cingulate gyrus, and thalamus..$^{7-9,49-52}$ Similar $\underline{A s}$ inte the spinal cord, brain atrophy starts to evolve within the first months after SCI and continues_-for at least ever the next twetwo years -post-injury[A: how many years?]. ${ }^{9,53}$ The resulting changes in tissue volume are clinically relevant. For example, greater volume reductions in the brainstem, during the first 6 months post-SCI were associated with poorer recovery of lower limb motor function. Interestingly, performance improvements due to intensive lower limb training in chronic SCI patients lead to volume increases within the atrophied brainstem, indicating reorganisation processes.${ }^{54}$ Likewise neuropathic pain intensity has been shown to be associated with reductions in primary sensory cortices and thalamus $^{49}$ as well as tincreases in grey matter volume within the anterior cingulate gyrus and primary motor cortices as well as reductions in primary sensory cortices and thalamus ${ }^{47}$ indicating reorganisation processes. ${ }^{55}$ 


\section{Advances in MRI technology}

-Conventional MRI, although sensitive to macrostructural cord and brain pathology, does not provide specific and quantitative microstructural measures of neurodegeneration and plasticity processes, making it difficult to draw specific conclusions about the underlying cause of the observed signal changes on T1- and T2-weigthed MRI scans. Thus, there is a pressing need to establish the missing link between measured MRI signals and changes in the underlying tissue microstructure and neurovascular function to explain and better understand the disease processes associated with SCI. Novel qMRI protocols of the spinal cord ${ }^{15,56}$ and brain ${ }^{17,57}$ have the potential to measure neural changes at the microstructural level. This is because the degree of myelination, iron content and neuronal microstructure are reflected in MR relaxation times, magnetisation transfer and diffusion of water molecules which can be measured at the voxel-level in the spinal cord ${ }^{15,56}$ and brain. ${ }^{17,57}$ qMRI aims at providing values comparable between individuals and they are specific to particular structural states, for example axonal degeneration or demyelination. ${ }^{56}[\mathrm{~A}$ : add supporting reference] Key stateof_-the--art methods such as XXrelaxometry mapping and diffusion MRI [A: please completel en qMRI-hastes been identified which hold-may have the potential to reveal the underlying pathophysiology after human SCI. ${ }^{56}$

The most common qMRI technique is diffusion-weighted imaging, which probes the directional diffusivity of water molecules and shows sensitivity and specificity to the axon and myelin pathology. ${ }^{58}$ Frequently, diffusion-imaging data are analyzedanalysed using a tensor model, i.e. applying diffusion tensor imaging (DTI) ${ }^{59}$ However, the tensor model makes several restricting assumptions, which complicate the interpretation of major DTI indices (i.e. fractional anisotropy, axial and radial diffusivity, or mean diffusivity) with respect to the underlying pathology. Novel biophysical models of diffusion contrast are being developed based on different mathematical models and could alleviate this issue, although 
these modelling approaches have yet to be validated; are being controversially discussed partially due to inconsistent and poerly understood outcomes and-acquisition/modelling variability versus biological variability. ${ }_{-}^{60}$ [A: could you briefly add why this is the case?]

The quantitative measurement of relaxation and magnetization transfer parameters has been

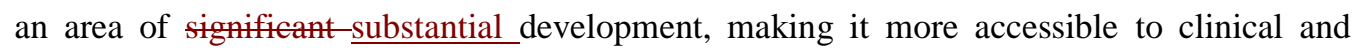
preclinical research applications. For example, the multi parametric mapping (MPM) approach combines different MRI modalities in one protocol quantifying MR parameter measures of magnetization transfer (MT), and longitudinal and effective transverse relaxation rates $(\mathrm{R} 1, \mathrm{R} 2 *)^{61}$ (Figure 4$)$. The link between these qMRI metrics and histology has previously-been studied testudied to probe the micro-structure of the human neocortex, focusing specifically on myelin, iron, and neuronal fibre mapping. in $x x x-$ [A: please complete]. ${ }^{57}$ MT measures correlate with histologically measured myelin content, ${ }^{62}$ whereas certain quantitative relaxation rate measurements correlate with iron content. ${ }^{63}$ These results $\underline{\text { may provide useful and specific biomarkers such as oligodendrocyte, glial cells, and iron rich }}$ $\underline{\text { fibres, with potential clinical impact in different pathologies, including SCI }}{ }^{57}$ [A: what does

\section{it mean clinically?]}

\section{Clinical qMRI studies}

Building on advances of qMRI methods, recent-studies in patients with SCI have focused on improving the detection and quantification of tissue-specific spinal cord and brain pathology —and on elucidating its relationship with clinical impairment. DTI applied to the white matter of the injured spinal cord demonstrates lower fractional anisotropy (FA) (sensitive to myelination, axon diameter, fiber density \& organization)-[A: fractional anisotropy? Explain importance of FA] values above and below the lesion, both in acute ${ }^{64}$ and chronic patients. $^{6,12}$ [A: please link sentences] For processing diffusion weighted images, the advent 
of a spinal cord template, ${ }^{65}$ and post-processing tools ${ }^{66,67}$ included in the spinal cord toolbox ${ }^{68}$ now offers the advent of a spinal cord template ${ }^{62}$, the spinal cord toolbox ${ }^{63}$ and post processing tools ${ }^{64,65}$ now offers the opportunity to assess tract-specific DTI changes at the voxel-level across the entire spinal cord. At both the cervical ${ }^{6}$ and lumbar enlargement ${ }^{48}$ DTI has shown tissue specific decreased fractional and axial diffusivities and increased radial diffusivities in the corticospinal tract and the dorsal columns. The former effects have been associated with axonal degeneration ${ }^{58}$ whereas the latter is associated with demyelination. ${ }^{58}$ The results are suggestive of retrograde and anterograde degeneration of descending motor pathways and ascending afferent spinal projections, respectively. Moreover, the grey matter of the lumbar enlargement featured decreased fractional and axial diffusivity, indicating trans-synaptic degeneration of motor neuron pools deprived of supraspinal input. ${ }^{48}$ DTI applied to the brain showed impaired microstructure along the cranial projection of the corticospinal tract, ${ }^{13,14}$ as well as other brain areas such as the corpus callosum, and fibre tracts such as [A: addition ok?] inferior and superior longitudinal fasciculi, and the inferior fronto-occipital fasciculus; ${ }^{11}$ suggesting large-scale structural degeneration and reorganization across the brain.

The MPM protocol ${ }^{61,69}$ (Figure 4), applied to acute SCI patients, revealed that spinal cord atrophy was paralleled by myelin-sensitive MT decreases, ${ }^{7}$ while in brain areas undergoing progressive atrophy, myelin content decreased and iron content increased. ${ }^{7-9}$ For example, the atrophying primary motor cortex showed lower myelin content (reflected by decreased MT and $\mathrm{R} 1^{8}$ ), while the atrophying thalamus showed iron deposition (reflected by increased $\left.\mathrm{R} 2 *^{8}\right)$. Moreover, within the cerebellum, accelerating atrophy was paralleled by a deceleration of myelin-sensitive MT. These bidirectional effects suggest the changes in myelination $^{62}$ and iron content $^{70}$ reflecting dynamic processes in the context of compensation, decompensation and the compounding of functional deficits. ${ }^{5}$
Formatted: Font: (Default) Times New Roman, 12 pt, Font color: Text 1 English (United Kingdom)

Formatted: Font: (Default) Times New Roman, 12 pt, Font color: Text English (United Kingdom)

Formatted: Font: (Default) Times New Roman, 12 pt, Font color: Text English (United Kingdom)

Comment [MS13]: Response R4.4 
Clinical recovery occurs most rapidly within the first six months and plateaus at approximately 2 years post-SCI. ${ }^{71}$ At present, intensive (hours of training daily within 6 months post injury [A: define intensive]neurorehabilitation is the only known means to improve functional recovery. Neurorehabilitation per se is believed to promote neurological changes such as cortical and spinal cord neural circuit reorganisation, which is assumed to translate into improved function. A few longitudinal qMRI studies within the range of one to two years post-SCI follow-up [A: how long were these?] in the sub acute 4 : how many months? I phase of injury ( $<2$ months post SCI $)$ have found that better ISNCSCI lower extremity motor score recovery assessed clinically using the International Standards for Neurological Classification of SCI protocol was predicted by less cervical eord atrophyspinal cord atrophy, ${ }^{9,10}$ and cord diffusion alterations. ${ }^{72}$ Early after SCI $(<2$ months post-injury $)\lceil$ A: how many months?]and at the level of the brain, greater ISNCSCI lower extremity motor recovery was associated with less cranial corticospinal tract atrophy. ${ }^{9}$ At the microstructural level, a worse ISNCSCI pin-prick score was associated with a greater increase in GM R2* in the thalamus, ${ }^{9}$ a better ISNCSCI lower extremity motor recovery was predicted by by an increase in R2* in the cerebellum ${ }^{9,10}$-a smaller [A: define small $]$ decrease in MT in the somatosensory cortex ${ }^{9}$ and a greater decrease $\mathrm{R} 2 *$ in the right cerebellum,,$\frac{10}{}$ and increased functional connectivity between primary motor cortex and supplementary motor and premotor cortices. ${ }^{46}$ More substantial grey matter atrophy in the cerebellum was associated with impaired light-touch sensation, ${ }^{10}$ while greater increases in neuropathic pain intensity were associated with more extensive microstructural changes (increased R2*) in the secondary sensory cortex, anterior cingulate cortex, and cerebellum. ${ }^{9}$

These longitudinal qMRI studies within a two-year follow-up point to three important and clinically relevant findings: (i) while clinical recovery levels off at two years post-SCI $[\mathrm{A}$ : 
time frame is not entirely clear from your arguments above, approximately 2 years? ],

progressive changes in macroscopic and microstructural markers continue; (ii) while macrostructrual changes slow down at the level of the spinal cord, both macroscopic and microstructural measures of neurodegeneration show sustained changes in the brain; (iii) the changes that have the greatest predictive validity in relation to clinical outcome appear to be those at the level of the spinal cord, brainstem and cortex (e.g. spinal cord atrophy, cranial CST atrophy, lower MT in the primary motor cortex) [A: could you be more specific?] over the first 6 months. ${ }^{9,10}$ [A: would be good to support it with some references]

Implication for clinical trials

Eurrently, Tt The primary endpoint of choice in SCI trials so far is an improvement in clinical outcome measures. However, neuroimaging biomarkers holdhave the potential to supplement these clinical measures as they are sensitive to neuronal changes even when they do not yeteven before they mayprior to their -translateione into obvious clinical benefit. Currently,

clinical trials employ conventional MRI (e.g. T2-weighted signal characteristics of the cord) (Table 1) to account for gross macrostructural changes at the lesion site in the spinal cord for example after stem cell interventions. ${ }^{73-75}$ However, signal intensity changes in conventional MRI do not correspond with the specific and quantitative measures of microstructural deficits (e.g. demyelination and axonal degeneration) (e.g. , XX). ${ }^{15}$ With potential treatments targeting repair of the injured spinal cord, it is imperative to improve clinical trial design and efficiency, optimise patient stratification in the context of disease heterogeneity and identify sensitive trial outcome measures.

Based on the advances in MRI, discussed earliersuch as high resolution-conventional MRI, advanced relaxometry mapping and diffusion MRI- XXX , the application of neuroimaging biomarkers for SCI trials, which combine conventional MRI and qMRI assessments, is now 
feasible. This requires measures sensitive to the earliest changes following injury, which are quantifiablequantifiable, and which capture neural damage and plasticity. As qMRI ${ }^{15,57,76}$ is sensitive to microstructural aspects of specific tissue classes $[$ A: could you give an example or two?] of the eentral nervous systemCNS (e.g. myelin; and axonal integrity, and iron

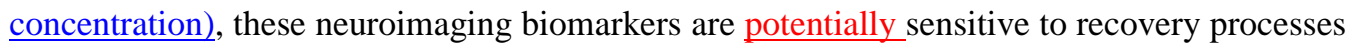
and treatment responses. ${ }^{15,17,57}$ Moreover, they bear the potential to provide short term surrogate end-points (i.e. changes over 6-12 months), which may reduce the time and cost associated with novel drug development. ${ }^{77,78}$ Despite a therapeutic intervention having an effect on imaging outcome such as halting atrophy, there is still some disconnect between changes in imaging outcomes and clinically meaningful recovery; the ultimate goal of a successful clinical trial. Thus, it may be useful to employ more than one imaging outcome in Comment [MOU20]: Response to E5 future trials to maximize understanding and interpretation of clinically meaningful findings.

Deploying advanced qMRI methods in multi-centre trials is challenging however, requiring high quality qMRI techniques_such as high field MR scanner (e.g. 3 Tesla), advanced $\underline{\text { software version and sophisticated image post-processing pipelinestoolbox tested- }[\mathrm{A} \text { : could }}$ you give an example or two to help with the flow?] to be implemented on the different scanner platforms from different manufacturers and different clinical sites across worldthe globe. Any resulting differences or performance issues may reduce the potential benefits for evaluating new therapies. Moreover, clinical trials usually run over years and hence scanner software and hardware upgrades as well as scanner replacements cannot always be avoided. Thus, there is a need to further improve intra-scanner and inter-scanner comparability of the qMRI protocols. The feasibility of combining multi-centre DTI data has been previously shown derived from 27 centres in xx countries with on using different 3T scanner models, software versions and pulse sequences has been shown_..$^{79,80}$ [A: add supporting ref]
[Ad 
However, critical parameters such as noise floor level and signal-to-noise floor ratio have to be monitored and adjusted to increase the statistical power estimates. ${ }^{79}$ [A: please link these highlighted sentences]Likewise, the MPM protocol was validated at 3T MRI scanner for use in multi-eentercentre studies based on custom-made ${ }^{61}$ and manufacturers based ${ }^{81}$ FLASH sequences. The same five volunteers were scanned at the three research sites in a travelling heads study design and demonstrated good comparability in another travelling heads study MPM protocols based on the manufacturers FLASH pulse sequences were assessed in five different clinical sites ${ }^{77}$ - Currently, the MPM protocol is being considered for a phase II multi-centre clinical trial (NISCI) (EudraCT: 2016-001227-31) investigating the neutralizing effects of an anti-Nogo-A antibody treatment for SCI. ${ }^{82}$ [A: if possible, please add NCT number] Thus, there is hope that effect sizes based on qMRI data may afford the opportunity to assess site-specific effects of intervention; essential for the translation of trial efficacy to clinical effectiveness. Hypothetical treatment effects, defined by slower longitudinal structural changes in these imaging measures, could be detectable over a realistic timescale $\underline{6}$ $\underline{\text { months post injury) [A: define realistic timescale] with potentially lower sample sizes }(<50}$ per treatment arm) than required for traditional clinical readouts. ${ }^{53}[\mathbf{A}$ : could you be more specific regarding the sample size?]

\section{Probing reorganization by means of Task specific and resting state functional MRI}

\section{and rs - FMRI}

Much of the discussion above concerned assessment of physical changes in the brain and spinal cord resulting from SCI and during recovery. Just as important is the ability to assess functional reorganization associated with SCI. Functional reorganization can be indirectly quantified, both in the brain and spinal cord, by means of fMRI that tracks task-dependent oxygen consumption that is indirectly related to neuronal activity (e.g. blood oxygen
Formatted: Font color: Text 1 , English (United Kingdom)

Comment [MS23]: Response to R3.2

Formatted: Font color: Text 1

Formatted: Font color: Text 1, English (United Kingdom)

Formatted: Font color: Text 1

Formatted: Font: 12 pt, Font color: Text 1

Formatted: Font: 12 pt, Font color: Text 1

Formatted: Font: 12 pt, Font color: Text 1

Formatted: Font: 12 pt, Font color:

Text 1

Formatted: Font: 12 pt, Font color: Text 1, English (United Kingdom)

Formatted: Font: 12 pt, Font color: Red, English (United Kingdom)

Formatted: Font: 12 pt, Font color: Red

Formatted: Font: 12 pt, Font color: Red

Formatted: Font: 12 pt, Font color: Red, English (United Kingdom)

Formatted: Font: 12 pt, Font color: Red, English (United Kingdom)

Comment [MS24]: We have not registered $\mathrm{NISCl}$ yet, work under progress. But we have EudraCT: 2016 001227-31 from The European Medicines Agency;: number adde into the text now.

Comment [WM(25]: Edit ok? 
dependent signal (BOLD)). In the absence of an explicit task, neuronal activity can also be studied by means of rs-fMRI analysis, which is based on low frequency spontaneous fluctuations in the BOLD signal._-rs-fMRI provides an indirect measurement of connectivity that allows for characterization of distinct functional networks in the brain or spinal cord. ${ }^{83}$

Motor and sensory recovery after SCI is associated with functional reorganization of the sensorimotor networks. ${ }^{84-86}$ fMRI studies after chronic SCI have inferred cortical reorganization through increased task-dependent activation in the primary motor cortex, cerebellum, and parietal lobe. ${ }^{84}$ Interestingly, in 23 complete (AIS A) a portion of SCI patients with complete impairment, in clinical terms (AIS A), stimulation below the level of the injury resulted in activation in the relevant somatosensory cortices. ${ }^{86}$ This suggests that preserved tissue bridges ${ }^{42}$ continue to carry functional information, but are insufficient to produce clinically meaningful activations or functions.

In a similar manner, s $\underline{S}$ pinal cord fMRI studies have also found that significant $\underline{\text { substantial }}$ [A: statistically?] task-related spinal activity, in response to stimuli are retained above and below the injury site. ${ }^{22-24}$ This suggests-_that next to cortical reorganizationthat,-the spinal cord is actively engaged in plastic processes that can result in recovery of function. Interestingly, this may also contribute to the emergences of neuropathic pain conditions ${ }^{85}$ which has been associated with maladaptive plasticity. ${ }^{49}$ [A: please elaborate on this as it might not be obvious for non-experts] Thus, spinal fMRI is feasible in the clinical setting ${ }^{87}$ and can identify changes in neural processing in relation to the location and extent of injury. Although the different-fMRI methods 4 : do you mean fMRI and rs-fMRI ? 1 that have been used-are readily available on clinical MR systems with a good spatial resolution, the analysis requires sophisticated post-processing tools ${ }^{68}$ and the interpretation of the functionally activated voxels remains challenging. ${ }^{16}$ [A: this is not also the case with DTI etc? $]$ Further advances in MRI hardware (sensitive MRI coils ${ }^{88}$ ), and in MRI software (optimized localized 
shimming ${ }^{89}$ ) and in other areas (eg, XXimage postprocessing) are expected to increase the value [A: for what? monitoring, biomarker?] of spinal cord fMRI as a biomarker in the near future for probing reorganization and plasticity induced by injury.

The application of rs-fMRI has gained momentum as it does not require an explicit task nor active participation of the individual. Both in acute ${ }^{19-21}$ and chronic ${ }^{90-92}$ SCI patients, connectivity changes can be observed across the motor system as well as in areas of cognitive control_(i.e. the bilateral dorsal anterior cingulate cortex dACC, dorsal lateral prefrontal cortex (DLPFC) and caudate) (eg, XX); with the magnitude of change, in both, relating to the recovery of function. ${ }^{19-21}$ Thus, connectivity changes in brain networks might reflect compensatory strategies to overcome functional deficit. However, rs-fMRI is an emerging field featuring a wide range of pre-processing and analytical approaches, which make it difficult to compare the outcomes of the different studies. Nevertheless, rs-fMRI holds promise as a more reliable and useful outcome measure in the clinical setting. Compared to conventional fMRI, rs-fMRI is easily applicable and does not depend on explicit tasks that require the attention and participation of the individual. To date - although technically feasible $^{25,83,93}$ there have been no rs-fMRI studies in patients with of the injured spinat eord $\underline{\mathrm{SCI}}$ conducted because it is challenging to obtain reliable rs-fMRI data from the spinal cord in SCI patients due to a number of technical issues; such as detrimentalthe influence of physiological noise and metallic implants. - [A: could you please speculate why this has been the case?] Nonetheless, results obtained in healthy controls have consistently shown robust networks with extensive connectivity between spinal cord regions as well as across the brainstem and the spinal cord. ${ }^{83,94}$ Based on these results, rs-fMRI of the injured spinal cord would be expected to demonstrate regions with altered/absent connectivity to other spinal cord regions as well as dynamic connectivity changes during recovery. This would allow to 
monitor functionally relevant changes within the spinal cord during the process of recovery.

This awaits validation.

\section{Conclusions and future directions}

Traumatic SCI causes permanent disability, and yet despite advances in medical management (eg,such as XXimproved rehabilitation cares and clinical assessments) [A: give an example

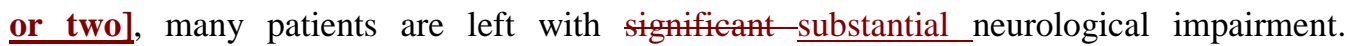
Currently, intensive care measures including blood pressure augmentation, neuroprotective approaches with anti-inflammatories, _neurosurgical decompression and stabilization and intensive neurorehabilitation are the only interventions applied to promote partial recovery. ${ }^{71}$ Understanding the pathophysiological sequelae would help tFo reduce and prevent disease burden and would facilitate, it is imperative to the development of effective regenerative and neuroprotective treatments. Understanding the pathophysiological sequelae (Figure 5, for summary overview) that eventually affect the entire neuroaxis is essential for drug development. In this review we emphasize that b[A: Please link sentences]

Both conventional MRI and qMRI of the spinal cord and brain can guide diagnostic workup, identify predictors of recovery, elucidate SCI pathogenesis and provide surrogate endpoints in future clinical trials. ${ }^{16,45}$ Conventional T2-weighted sagittal and axial MRI are key methods to identify the extent of the intramedullary injury ${ }^{27,28}$ and to identify prognostic parameters such as intramedullary lesion length and preserved midsagittal tissue bridges. ${ }^{37,42,44}$ Advanced qMRI sequences, such as DTI and MPM, applied remotely from the injury can identify microstructural changes such as axonal degeneration, demyelination, and iron deposition across the entire neuroaxisneuraxis ${ }^{15,9}$. [A: add supporting reference $[$ Combinations of serial conventional MRI and qMRI represent key modalities (Table 2)-for a better assessment [A: compared to what?] of spinal cord function compared to clinical assessment, and
Comment [WM(31]: what would eb the clinical implications?

Comment [WM(32]: It should be made clear here and in the intro (something along these lines) that until recently, past clinical markers (AIS and AMS) were the only variable used as predictors of outcome following spinal cord injury. Now structural MRI imaging markers such as the Basic Score and intramedullary lesion length (IMLL) were considered as useful predictors of outcome. Based on your review is seems that the future belongs to better understanding qualitative MRI (qMRI: magnetization transfer, relaxation maps and diffusion characteristics) and using it as an indicator of outcome.

Comment [MS33]: Response to R2.5

Formatted: Font: 12 pt, Bold, Font color: Red

Formatted: Font: 12 pt, Bold, Font color: Red

Formatted: Font: 12 pt, Font color: Red

Comment [MS34]: Response to R2.4 
further, in elucidating the relationship between clinical impairment and remote secondary changes in the spinal cord and brain. In addition, these quantifiable changes appear to have notable predictive validity, rendering them viable outcomes for interventional trials. ${ }^{9,53}$

The advances reviewed in this paper suggest On the basis of the studies included in this review, wWe believe recommend that neuroimaging of the spinal cord should be routinely performed as a routinein clinical practice in clinical routine and in interventional trials? in a number of instances (Table 2). Conventional MRI should include both sagittal and axial views to assess the level and extent of injury within the first 48 hours (e.g. BASIC score). These scans should be repeated 3-4 weeks later to quantify the dynamics of intramedullary lesion length and to identify the amount of preserved midsagittal tissue bridges. To investigate pathophysiological changes in the research setting, we recommend employing qMRI methods, such as DTI and MPM, should ebbe used as these can probe microstructural changes of the spinal cord and brain. Neuroimaging outcome measures derived from both conventional MRI and qMRI protocols should be considered as they as they are predictors of recovery. ${ }^{9,53}$ However, a careful evaluation of the variance caused by differences between scanners and an assessment of reproducibility is required, adding to the complexity of multicentre trials.

Current understanding of trauma induced changes across the heuroaxisneuraxis remains incomplete_(Figure 5). A key requirement to assessing plasticity in vivo is ultra-high spatial resolution on the order of hundreds of microns. The -To visualize and quantify ultra-scale tissue properties of grey and white matter ean now be assessed using biophysical models that exploit symmetries in the organization of microstructure are required.- Emerging However, technological and imaging developments-are required, at higher field strengths (e.g. 7T MRI 
scanner), such as improvements in RF coil designs, pulse-sequence design, improved localized magnetic field shimming methods, ${ }^{89}$ suppression of MRI artefacts induced by orthopaedic implants, ${ }^{33}$ and changes in data sampling schemes ${ }^{56}$ will provide the necessary means for these. Further, the development of new biophysical models inlinking the MRI measurements mechanistically with the underlying microstructure are is a critical area for future research. These models can combine multiple different MRI contrasts with different views on the underlying microstructure to address the intractable problem of accurately making inferences concerning the microstructure from single contrasts. This was recently done, fFor example, modelling the relative myelination of axons (e.g. g-ratio mapping ${ }^{95,96}$ ). The integration and unifying across the different contrasts and spatial scales (from micrometersmicrometres to eentimeterscentimetres) is the object of intensive and ongoing research in the MRI community. ${ }^{15,57}$ However, MRI contrasts remain indirect measures of changes in the microstructure and composition of the tissue. Therefore, knowledge about the underlying changes is needed for interpretation of the non-invasive qMRI data and for improving the biophysical models. qMRI data will need to undergo histological validation (cross-validation of the MPM contrasts is currently performed in aan multiit-national $\begin{array}{llll}\text { ERANET funded } \quad \text { "hMRIofSCI consortium } & \text { (https://www.neuron- }\end{array}$ eranet.eu/ media/hMRIofSCI.pdf)) of tissue samples from experimental SCI models in order to shed light on the mechanistic underpinnings of changes observed with different MRI contrasts. Finally, multicentre and longitudinal studies, with large patient cohorts that employ qMRI of the spinal cord and brain would be useful to better characterize primary and secondary disease changes, along with their dynamics, and also support and extent current mono-centric and mainly cross-sectional studies. Increasing our understanding of the $\underline{\text { sequelae after SCI will allow eventually to predict individual trajectories of recovery [A: }}$

\section{please link to patient outcome]}

Comment [WM(41]: Please revise fo

clarity

Comment [WM(42]: More

information on this consortium migh be beneficial

Comment [WM(43]: This part could be streamlined as revised as not very easy to follow for non-experts 


\section{Acknowledgment}

We thank Dr Joshua Grant for proof reading. MGF is supported by grants from the Canadian Institute of Health Research (CIHR), AOSpine, Wings for Life Foundation, Craig Neilsen Foundation and the International Spinal Research Trust (ISRT). He is also-supported by the Halbert Chair in Neural Repair and Regeneration and the Dezwirek Foundation. K. F. is funded by a Wellcome Trust Principal Research Fellowship (Ref: 088130/Z/09/Z). A.J.T. acknowledges support from the UCL/UCLH NHHR Biomedical Research Centre.

\section{Author's contribution}

$\mathrm{PF}, \mathrm{MS}, \mathrm{NW}, \mathrm{KF}, \mathrm{MF}, \mathrm{AT}$ and AC contributed to the literature search, data interpretation and writing of the manuscript. PF and MS created the figures and tables.

\section{Search strategy and selection criteria}

We conducted a MEDLINE search focused on traumatic SCI using PubMed including only English language publications from 01.2013 until 101.20198. The search headings included the following words "traumatic spinal cord injury" in combination with search terms “atrophy”, “demyelination" “diffusion” conventional MRI”, “quantitative MRI”, | "neurodegeneration", clinical trial", "longitudinal", and "MRI prediction". Further articles were identified by searching the list of references cited in the manuscripts that were reviewed. The final reference list was generated on the basis of relevance to the topics covered in this Review.

\section{Declaration of interests}

MGF is supported by grants from the Canadian Institute of Health Research (CIHR), Formatted: Level 1 
Research Trust (ISRT). He is also supported by the Halbert Chair in Neural Repair and

Regeneration and the Dezwirek Foundation. K. F. is funded by a Welleome Trust Principat

Research Fellowship (Ref: 088130/Z/09/Z).

A.J.T. acknowledges support from the UCL/UCLHNHHR Biomedical Research Centre.

Patrick Freund reports grants from ERA-NET NEURON (hMRIofSCI no: 32NE30_173678), grants from NISCI supported by the European Union's Horizon 2020 research and innovation program under the grant agreement No 681094, and supported by the Swiss State Secretariat for Education, Research and Innovation (SERI) under contract number 15.0137, grants from Wings for life charity (No WFL-CH-007/14), grants from International Foundation for Research (IRP-158), during the conduct of the study.

Maryam Seif has nothing to disclose.

Nikolaus Weiskopf reports grants from European Research Council / ERC grant agreement $\mathrm{n}^{\circ}$ 616905, grants from BMBF (01EW1711A B) in the framework of ERA-NET NEURON, grants from BRAINTRAIN European research network (Collaborative Project) supported by the European Commission (Grant agreement $n^{\circ}$ 602186), grants from NISCI supported by the European Union's Horizon 2020 research and innovation program under the grant agreement No 681094, and supported by the Swiss State Secretariat for Education, Research and Innovation (SERI) under contract number 15.0137, grants from UCL Impact Awards and Siemens Healthcare, during the conduct of the study; _and The Wellcome Centre for Human Neuroimaging and Max Planck Institute for Human Cognitive and Brain Sciences have institutional research agreements with Siemens Healthcare. He is also Associate Editor of Frontiers in Brain Imaging Methods.

Comment [WM(46]: Not declared in the ICMJE form; please submit amend form

Comment [MS47]: Now declared 
Michael Fehlings is chair of the scientific advisory board of Fortuna Fix. MF is supported by grants from the Canadian Institute of Health Research (CIHR), AOSpine, Wings for Life Foundation, Craig Neilsen Foundation and the International Spinal Research Trust (ISRT). $\underline{\text { He is also supported by the Halbert Chair in Neural Repair and Regeneration and the }}$ Dezwirek Foundation.

Karl Friston is funded by a Wellcome Trust Principal Research Fellowship (Ref: $\underline{088130 / \mathrm{Z} / 09 / \mathrm{Z})}$. has nothing to disclose.

Alan Thompson reports personal fees paid to his institution andotherfrom Eisai Ltd and fees and support for travel from Hoffmann-La Roche outside the submitted work; -and Editorial Board member, The Lancet Neurology, receiving free subscription; Editor-in-Chief, Multiple Sclerosis Journal, honorarium from SAGE Publications; support for travel as Chair, Scientific Advisory Committee, International Progressive MS Alliance, and member, National MS Society (USA), Research Programs Advisory Committee. Received honoraria and support for travel for lecturing from EXCEMED and Almirall. A.J.T. acknowledges also support from the UCL/UCLH NIHR Biomedical Research Centre.

Armin Curt reports grants from European Union's Horizon 2020 No 681094, grants from Swiss State Secretariat for Education (No 15.0255), grants from ERA-NET NEURON grant (SILENCE - no: 31NE30_173667), grants from ERA-NET NEURON grant (hMRIofSCI- no: 
32NE30_173678), grants from Swiss National Science Foundation (Pain control systems no. 320030_169250), during the conduct of the study. 


\section{References}

1 Ellingson BM, Salamon N, Holly LT. Imaging techniques in spinal cord injury. World Neurosurg 2014; 82: $1351-8$

2 Kumar Y, Hayashi D. Role of magnetic resonance imaging in acute spinal trauma: a pictorial review. BMC Musculoskelet Disord 2016; 17: 310.

3 Shah LM, Ross JS. Imaging of Spine Trauma. Neurosurgery 2016; 79: 626-42.

4 Fehlings MG, Martin AR, Tetreault LA, et al. A Clinical Practice Guideline for the Management of Patients With Acute Spinal Cord Injury: Recommendations on the Role of Baseline Magnetic Resonance Imaging in Clinical Decision Making and Outcome Prediction. Glob Spine J 2017; 7: 221S $230 \mathrm{~S}$.

5 Freund P, Friston K, Thompson AJAJ, et al. Embodied neurology: an integrative framework for neurological disorders. Brain 2016; 139: 1855-61.

6 Huber E, David G, Thompson AJ, Weiskopf N, Mohammadi S, Freund P. Dorsal and ventral horn atrophy is associated with clinical outcome after spinal cord injury. Neurology 2018; 90: 10.1212/WNL.0000000000005361.

7 Grabher P, Callaghan MF, Ashburner J, et al. Tracking sensory system atrophy and outcome prediction in spinal cord injury. Ann Neurol 2015; 78: 751-61.

8 Freund P, Weiskopf N, Ashburner J, et al. MRI investigation of the sensorimotor cortex and the corticospinal tract after acute spinal cord injury: a prospective longitudinal study. Lancet Neurol 2013; 12: $873-81$.

9 Ziegler G, Grabher P, Thompson AJ, et al. Progressive neurodegeneration following spinal cord injury: Implications for clinical trials. Neurology 2018; 90: e1257-66.

10 Seif M, Curt A, Thompson AJ, Grabher P, Weiskopf N, Freund P. Quantitative MRI of rostral spinal cord and brain regions is predictive of functional recovery in acute Spinal cord injury. NeuroImage Clin 2018; published online Aug 19. DOI:10.1016/J.NICL.2018.08.026.

11 Ilvesmäki T, Koskinen E, Brander A, Luoto T, Öhman J, Eskola H. Spinal cord injury induces widespread chronic changes in cerebral white matter. Hum Brain Mapp 2017; 3647: 3637-47.

12 Koskinen E, Brander A, Hakulinen U, et al. Assessing the State of Chronic Spinal Cord Injury Using Diffusion Tensor Imaging. J Neurotrauma 2013; 30: 1587-95.

13 Koskinen EA, Hakulinen U, Brander AE, Luoto TM, Ylinen A, Öhman JE. Clinical correlates of cerebral diffusion tensor imaging findings in chronic traumatic spinal cord injury. Spinal Cord 2014; 52 : 202-8.

14 Jirjis MB, Vedantam A, Budde MD, Kalinosky B, Kurpad SN, Schmit BD. Severity of spinal cord injury influences diffusion tensor imaging of the brain. J Magn Reson Imaging 2016; 43: 63-74.

15 Cohen-Adad J. Microstructural imaging in the spinal cord and validation strategies. Neuroimage 2018; published online April 7. DOI:10.1016/j.neuroimage.2018.04.009.

16 Cadotte DW, Akbar MA, Fehlings MG, Stroman PW, Cohen-Adad J. What Has Been Learned from Magnetic Resonance Imaging Examination of the Injured Human Spinal Cord: A Canadian Perspective. J Neurotrauma 2018; 35: 1942-57.

17 Trampel R, Bazin PL, Pine K, Weiskopf N. In-vivo magnetic resonance imaging (MRI) of laminae in the human cortex. Neuroimage. 2017; published online Sept 20. DOI:10.1016/j.neuroimage.2017.09.037.

18 Lerch JP, van der Kouwe AJW, Raznahan A, et al. Studying neuroanatomy using MRI. Nat Neurosci 2017; 20: 314-26.

19 Hou J-M, Sun T-S, Xiang Z-M, et al. Alterations of resting-state regional and network-level neural function after acute spinal cord injury. Neuroscience 2014; 277: 446-54.

20 Zhu L, Wu G, Zhou X, Li J, Wen Z, Lin F. Altered Spontaneous Brain Activity in Patients with Acute Spinal Cord Injury Revealed by Resting-State Functional MRI. PLoS One 2015; 10: e0118816.

21 Hawasli AH, Rutlin J, Roland JL, et al. Spinal Cord Injury Disrupts Resting-State Networks in the Human Brain. J Neurotrauma 2018; 35: 864-73.

22 Stroman PW, Khan HS, Bosma RL, et al. Changes in Pain Processing in the Spinal Cord and Brainstem after Spinal Cord Injury Characterized by Functional Magnetic Resonance Imaging. J Neurotrauma 2016; 33: 1450-60.

23 Zhong X-P, Chen Y-X, Li Z-Y, Shen Z-W, Kong K-M, Wu R-H. Cervical spinal functional magnetic resonance imaging of the spinal cord injured patient during electrical stimulation. Eur Spine J 2017; 26: 71-7.

24 Alexander M, Kozyrev N, Figley CR, Richards JS. Altered spinal cord activity during sexual stimulation in women with SCI: a pilot fMRI study. Spinal Cord Ser Cases 2017; 3: 16041. 
25 Barry RL, Conrad BN, Smith SA, Gore JC. A practical protocol for measurements of spinal cord functional connectivity. Sci Rep 2018; 8: 16512.

26 Kirshblum SC, Waring W, Biering-Sorensen F, et al. Reference for the 2011 revision of the International Standards for Neurological Classification of Spinal Cord Injury. J Spinal Cord Med 2011; 34: $547-54$.

27 Talbott JF, Whetstone WD, Readdy WJ, et al. The Brain and Spinal Injury Center score: a novel, simple, and reproducible method for assessing the severity of acute cervical spinal cord injury with axial T2-weighted MRI findings. J Neurosurg Spine 2015; 23: 1-10.

28 Aarabi B, Sansur CA, Ibrahimi DM, et al. Intramedullary Lesion Length on Postoperative Magnetic Resonance Imaging is a Strong Predictor of ASIA Impairment Scale Grade Conversion Following Decompressive Surgery in Cervical Spinal Cord Injury. Neurosurgery 2017; 80: 610-20.

29 Jungmann PM, Agten CA, Pfirrmann CW, Sutter R. Advances in MRI around metal. J Magn Reson Imaging 2017; 46: 972-91.

30 Sundseth J, Jacobsen EA, Kolstad F, Nygaard OP, Zwart JA, Hol PK. Magnetic resonance imaging evaluation after implantation of a titanium cervical disc prosthesis: a comparison of 1.5 and 3 Tesla magnet strength. Eur Spine J 2013; 22: 2296-302.

31 Lee YH, Lim D, Kim E, Kim S, Song H-T, Suh J-S. Feasibility of fat-saturated T2-weighted magnetic resonance imaging with slice encoding for metal artifact correction (SEMAC) at 3T. Magn Reson Imaging 2014; 32: 1001-5.

32 Song KD, Yoon YC, Park J. Reducing metallic artefacts in post-operative spinal imaging: slice encoding for metal artefact correction with dual-source parallel radiofrequency excitation MRI at 3.0 T. Br J Radiol 2013; 86: 20120524.

33 Worters PW, Sung K, Stevens KJ, Koch KM, Hargreaves BA. Compressed-sensing multispectral imaging of the postoperative spine. J Magn Reson Imaging 2013; 37: 243-8.

34 Dalkilic T, Fallah N, Noonan VK, et al. Predicting Injury Severity and Neurological Recovery after Acute Cervical Spinal Cord Injury: A Comparison of Cerebrospinal Fluid and Magnetic Resonance Imaging Biomarkers. J Neurotrauma 2018; 35: 435-45.

35 Martínez-Pérez R, Cepeda S, Paredes I, Alen JF, Lagares A. MRI Prognostication Factors in the Setting of Cervical Spinal Cord Injury Secondary to Trauma. World Neurosurg 2017; 101: 623-32.

36 Matsushita A, Maeda T, Mori E, et al. Subacute T1-low intensity area reflects neurological prognosis for patients with cervical spinal cord injury without major bone injury. Spinal Cord 2016; 54: 24-8.

37 Le E, Aarabi B, Hersh DS, et al. Predictors of intramedullary lesion expansion rate on MR images of patients with subaxial spinal cord injury. J Neurosurg Spine 2015; 22: 611-21.

38 Farhadi HF, Kukreja S, Minnema A, et al. Impact of Admission Imaging Findings on Neurological Outcomes in Acute Cervical Traumatic Spinal Cord Injury. J Neurotrauma 2018; 35: 1398-406.

39 Aarabi B, Olexa J, Chryssikos T, et al. Extent of Spinal Cord Decompression in Motor Complete (American Spinal Injury Association Impairment Scale Grades A and B) Traumatic Spinal Cord Injury Patients: Post-Operative Magnetic Resonance Imaging Analysis of Standard Operative Approaches. $\boldsymbol{J}$ Neurotrauma 2018; : neu.2018.5834.

40 Haefeli J, Mabray MC, Whetstone WD, et al. Multivariate Analysis of MRI Biomarkers for Predicting Neurologic Impairment in Cervical Spinal Cord Injury. Am J Neuroradiol 2017; 38: 648-55.

41 Mabray MC, Talbott JF, Whetstone WD, et al. Multidimensional Analysis of Magnetic Resonance Imaging Predicts Early Impairment in Thoracic and Thoracolumbar Spinal Cord Injury. J Neurotrauma 2016; 33: 954-62.

42 Huber E, Lachappelle P, Sutter R, Curt A, Freund P. Are midsagittal tissue bridges predictive of outcome after cervical spinal cord injury? Ann Neurol 2017; 81: 740-8.

43 Dhall SS, Haefeli J, Talbott JF, et al. Motor Evoked Potentials Correlate With Magnetic Resonance Imaging and Early Recovery After Acute Spinal Cord Injury. Neurosurgery 2018; 82: 870-6.

44 O'Dell DR, Weber KA, Berliner JC, et al. Midsagittal tissue bridges are associated with walking ability in incomplete spinal cord injury: A magnetic resonance imaging case series. J Spinal Cord Med 2018; : $1-4$.

45 Farhadi HF, Minnema AJ, Talbott JF, Aarabi B. Letter to the Editor: What Has Been Learned from Magnetic Resonance Imaging Examination of the Injured Human Spinal Cord: A Canadian Perspective. J Neurotrauma 2018; : neu.2018.6135.

46 Hou J, Xiang Z, Yan R, et al. Motor recovery at 6 months after admission is related to structural and functional reorganization of the spine and brain in patients with spinal cord injury. Hum Brain Mapp 2016; 37: 2195-209.

47 Prados F, Ashburner J, Blaiotta C, et al. Spinal cord grey matter segmentation challenge. Neuroimage 2017; 152: 312-29.

48 David G, Seif M, Huber E, et al. In vivo evidence of remote neural degeneration in the lumbar 
enlargement after cervical injury. Neurology 2019; : 10.1212/WNL.0000000000007137.

49 Jutzeler CR, Huber E, Callaghan MF, et al. Association of pain and CNS structural changes after spinal cord injury. Sci Rep 2016; 6: 18534.

50 Chen Q, Zheng W, Chen X, et al. Brain Gray Matter Atrophy after Spinal Cord Injury: A Voxel-Based Morphometry Study. Front Hum Neurosci 2017; 11: 211.

51 Grabher P, Blaiotta C, Ashburner J, Freund P. Relationship between brainstem neurodegeneration and clinical impairment in traumatic spinal cord injury. NeuroImage Clin 2017; 15: 494-501.

52 Mole TB, MacIver K, Sluming V, Ridgway GR, Nurmikko TJ. Specific brain morphometric changes in spinal cord injury with and without neuropathic pain. Elsevier 2014; 5: 28-35.

53 Seif M, Ziegler G, Freund P. Progressive ventricles enlargement and CSF volume increases as a marker of neurodegeneration in SCI patients : A longitudinal MRI study. In: Ismrm. 2017: 2381.

54 Villiger M, Grabher P, Hepp-Reymond M-C, et al. Relationship between structural brainstem and brain plasticity and lower-limb training in spinal cord injury: a longitudinal pilot study. Front Hum Neurosci 2015; 9: 254.

55 Jutzeler CR, Freund P, Huber E, Curt A, Kramer JLK. Neuropathic Pain and Functional Reorganization in the Primary Sensorimotor Cortex after Spinal Cord Injury. J Pain 2015; 16. DOI:10.1016/j.jpain.2015.08.008.

56 Stroman PW, Wheeler-Kingshott C, Bacon M, et al. The current state-of-the-art of spinal cord imaging: Methods. Neuroimage 2014; 84: 1070-81.

57 Edwards LJ, Kirilina E, Mohammadi S, Weiskopf N. Microstructural imaging of human neocortex in vivo. Neuroimage 2018; 182: 184-206.

58 Brennan FH, Cowin GJ, Kurniawan ND, Ruitenberg MJ. Longitudinal assessment of white matter pathology in the injured mouse spinal cord through ultra-high field (16.4T) in vivo diffusion tensor imaging. Neuroimage 2013; 82: 574-85.

59 Vedantam A, Jirjis MB, Schmit BD, Wang MC, Ulmer JL, Kurpad SN. Diffusion tensor imaging of the spinal cord: insights from animal and human studies. Neurosurgery 2014; 74: 1-8; discussion 8; quiz 8.

60 Novikov DS, Kiselev VG, Jespersen SN. On modeling. Magn Reson Med 2018; 79: 3172-93.

61 Lutti A, Bullmore ET, Weiskopf N, et al. Quantitative multi-parameter mapping of R1, PD*, MT, and R2* at 3T: a multi-center validation. Front Neurosci 2013; 7: 95.

62 Wang F, Li K, Mishra A, Gochberg D, Min Chen L, Gore JC. Longitudinal assessment of spinal cord injuries in nonhuman primates with quantitative magnetization transfer. Magn Reson Med 2016; 75: $1685-96$.

63 Langkammer C, Krebs N, Goessler W, et al. Quantitative MR Imaging of Brain Iron: A Postmortem Validation Study 1. Radiology 2010; 257: 455-62.

64 Vedantam A, Eckardt G, Wang MC, Schmit BD, Kurpad SN. Clinical Correlates of High Cervical Fractional Anisotropy in Acute Cervical Spinal Cord Injury. World Neurosurg 2015; 83: 824-8.

65 De Leener B, Fonov VS, Collins DL, Callot V, Stikov N, Cohen-Adad J. PAM50: Unbiased multimodal template of the brainstem and spinal cord aligned with the ICBM152 space. Neuroimage 2018; 165: $170-9$.

66 David G, Freund P, Mohammadi S. The efficiency of retrospective artifact correction methods in improving the statistical power of between-group differences in spinal cord DTI. Neuroimage 2017; 158: $296-307$.

67 Mohammadi S, Freund P, Feiweier T, Curt A, Weiskopf N. The impact of post-processing on spinal cord diffusion tensor imaging. Neuroimage 2013; 70: 377-85.

68 De Leener B, Lévy S, Dupont SM, et al. SCT: Spinal Cord Toolbox, an open-source software for processing spinal cord MRI data. Neuroimage 2017; 145: 24-43.

69 Callaghan MF, Freund P, Draganski B, et al. Widespread age-related differences in the human brain microstructure revealed by quantitative magnetic resonance imaging. Neurobiol Aging 2014; 35: 1862 72 .

70 Sauerbeck A, Schonberg DL, Laws JL, McTigue DM. Systemic iron chelation results in limited functional and histological recovery after traumatic spinal cord injury in rats. Exp Neurol 2013; 248: 53-61.

$71 \quad$ Ahuja CS, Wilson JR, Nori S, et al. Traumatic spinal cord injury. Nat Rev Dis Prim 2017; 3: 17018.

72 Shanmuganathan K, Zhuo J, Chen HH, et al. Diffusion Tensor Imaging Parameter Obtained during Acute Blunt Cervical Spinal Cord Injury in Predicting Long-Term Outcome. J Neurotrauma 2017; 34: 2964-71.

73 Hur JW, Cho T-H, Park D-H, Lee J-B, Park J-Y, Chung Y-G. Intrathecal transplantation of autologous adipose-derived mesenchymal stem cells for treating spinal cord injury: A human trial. J Spinal Cord Med 2016; 39: 655-64.

74 Mendonça MV, Larocca T, de Freitas Souza B, et al. Safety and neurological assessments after 
autologous transplantation of bone marrow mesenchymal stem cells in subjects with chronic spinal cord injury. Stem Cell Res Ther 2014; 5: 126.

75 Anderson KD, Guest JD, Dietrich WD, et al. Safety of Autologous Human Schwann Cell

Transplantation in Subacute Thoracic Spinal Cord Injury. J Neurotrauma 2017; 34: neu.2016.4895.

76 Zhou X, Sakaie KE, Debbins JP, et al. Quantitative quality assurance in a multicenter HARDI clinical trial at 3T. Magn Reson Imaging 2017; 35: 81-90.

77 Martin AR, Aleksanderek I, Cohen-Adad J, et al. Translating state-of-the-art spinal cord MRI techniques to clinical use: A systematic review of clinical studies utilizing DTI, MT, MWF, MRS, and fMRI. NeuroImage Clin 2016; 10: 192-238.

78 Cadotte DW, Fehlings MG. Will imaging biomarkers transform spinal cord injury trials? Lancet Neurol 2013; 12: 843-4.

79 Zhou X, Sakaie KE, Debbins JP, Narayanan S, Fox RJ, Lowe MJ. Scan-rescan repeatability and crossscanner comparability of DTI metrics in healthy subjects in the SPRINT-MS multicenter trial. Magn Reson Imaging 2018; 53: 105-11.

80 Samson RS, Lévy S, Schneider T, et al. ZOOM or Non-ZOOM? Assessing spinal cord diffusion tensor imaging protocols for multi-centre studies. PLoS One 2016; 11: e0155557.

81 Seif M, Leutritz T, Samson RS, et al. A multi-center study on fast full-brain quantitative multiparameter mapping of R1, MT, and R2*: scan-rescan repeatability and inter-site reproducibility. In: ISMRM. 2018: 1119.

82 Kucher K, Johns D, Maier D, et al. First-in-Man Intrathecal Application of Neurite Growth-Promoting Anti-Nogo-A Antibodies in Acute Spinal Cord Injury. Neurorehabil Neural Repair 2018; 32: 578-89.

83 Eippert F, Kong Y, Winkler AM, et al. Investigating resting-state functional connectivity in the cervical spinal cord at 3T. Neuroimage 2017; 147: 589-601.

84 Solstrand Dahlberg L, Becerra L, Borsook D, Linnman C. Brain changes after spinal cord injury, a quantitative meta-analysis and review. Neurosci Biobehav Rev 2018; 90: 272-93.

85 Jutzeler CR, Curt A, Kramer JLK. Relationship between chronic pain and brain reorganization after deafferentation: A systematic review of functional MRI findings. NeuroImage Clin 2015; 9: 599-606.

86 Wrigley PJ, Siddall PJ, Gustin SM. New evidence for preserved somatosensory pathways in complete spinal cord injury: A fMRI study. Hum Brain Mapp 2018; 39: 588-98.

87 Powers JM, Ioachim G, Stroman PW. Ten key insights into the use of spinal cord fmri. Brain Sci. 2018; 8: 173 .

88 Zhao W, Cohen-Adad J, Polimeni JR, et al. Nineteen-channel receive array and four-channel transmit array coil for cervical spinal cord imaging at 7T. Magn Reson Med 2014; 72: 291-300.

89 Finsterbusch J, Sprenger C, Büchel C. Combined T2*-weighted measurements of the human brain and cervical spinal cord with a dynamic shim update. Neuroimage 2013; 79: 153-61.

90 Min Y-S, Park JW, Jin SU, et al. Alteration of Resting-State Brain Sensorimotor Connectivity following Spinal Cord Injury: A Resting-State Functional Magnetic Resonance Imaging Study. J Neurotrauma 2015; 32: 1422-7.

91 Oni-Orisan A, Kaushal M, Li W, et al. Alterations in Cortical Sensorimotor Connectivity following Complete Cervical Spinal Cord Injury: A Prospective Resting-State fMRI Study. PLoS One 2016; 11: $\mathrm{e} 0150351$.

92 Kaushal M, Oni-Orisan A, Chen G, et al. Evaluation of whole-brain resting-state functional connectivity in spinal cord injury - a large-scale network analysis using network based statistic. J Neurotrauma 2016; : neu.2016.4649.

93 Barry RL, Smith SA, Dula AN, Gore JC. Resting state functional connectivity in the human spinal cord. Elife 2014; 2014: 1-15.

94 Sprenger C, Finsterbusch J, Buchel C. Spinal Cord-Midbrain Functional Connectivity Is Related to Perceived Pain Intensity: A Combined Spino-Cortical fMRI Study. J Neurosci 2015; 35: 4248-57.

95 Duval T, Lévy S, Stikov N, et al. g-Ratio weighted imaging of the human spinal cord in vivo. Neuroimage 2017; 145: 11-23.

96 Mohammadi S, Carey D, Dick F, et al. Whole-brain in-vivo measurements of the axonal G-ratio in a group of 37 healthy volunteers. Front Neurosci 2015; 9: 1-13.

97 Morawski M, Kirilina E, Scherf N, et al. Developing 3D microscopy with CLARITY on human brain tissue: Towards a tool for informing and validating MRI-based histology. Neuroimage 2018; 182: $417-$ 28.

98 Liewald D, Miller R, Logothetis N, Wagner H-J, Schüz A. Distribution of axon diameters in cortical white matter: an electron-microscopic study on three human brains and a macaque. Biol Cybern 2014; 108: $541-57$.

99 Haefeli J, Mabray MCC, Whetstone WDD, et al. No Title. 2017; 38: 648-55.

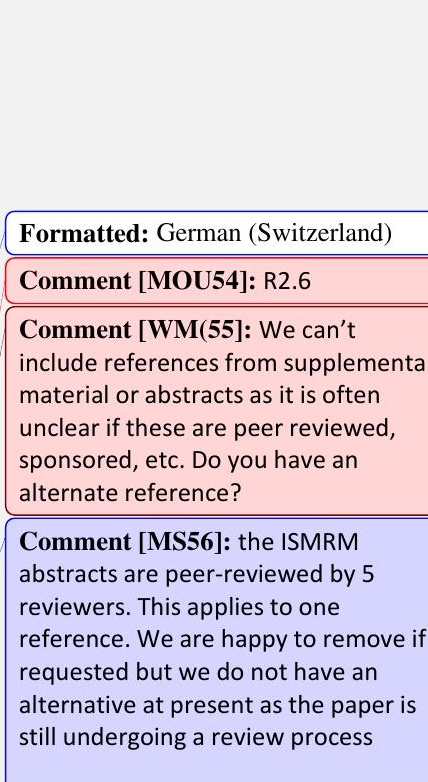

Formatted: German (Switzerland)

Formatted: German (Switzerland)

Formatted: German (Switzerland)

Formatted: German (Switzerland) 


\section{Figure captions}

\section{Figure 1: The BASIC score}

The BASIC score of SCIs comprises a 5-point ordinal MRI score for classifying acute SCIs on the basis of conventional axial T2-weighted scans. The BASIC score stratifies SCI according to the extent of transverse T2-weighted signal abnormality during the acute phase of the injury. Cartoon schematics (A), representative axial T2-weighted MRI scans (B), 3Dcolor surface plots based on the axial T2-weighted MRI (C), and brief definitions (D) for each of the 5 BASIC scores (ranging from 0 to 4). In the representative MRI scans (B), the external contour of the spinal cord is outlined in yellow for better delineation. (Reprinted from Talbott et al., $2015_{-}^{27}$ )

\section{Figure 2: Extent of lesion and tissue bridges}

Structural magnetic resonance imaging (MRI) indices assessed at the lesion site. A: Preoperative-Postoperative T2-weighted MRI of the cervical cord from a 17 year old 17 -yearold male patient with incomplete SCI patient_with traumatic spinal cord injury (male, ASIA Impairment Scale (AIS) AIS grade of A (complete)). Postoperative MRI at 32.5 hours after operation indicates an intramedullary lesion length (IMLL) of $102 \mathrm{~mm}$ (long bracket) with bleeding (short bracket) and myelomalacia (dotted lines) at the injury epicentre_-(Reprinted from Aarabi Bizhan et al., 2017 ${ }^{28}$ with permission from American Association for the Advancement of Science). (B: Demonstration of the lesion segmentation using mid-sagittal T2-weighted MRI within the cervical cord of a 51 year-old incomplete SCI patient (male, tetraplegic, AIS grade of $\mathrm{C}$ and with scan finding of central T2-weighted hyperintensive cord defect at the C6/C7 level). C: Demonstration of the lesion segmentation using mid-sagittal T2-weighted MRI within the thoracic cord of an 80 year old 10 -year-old incomplete SCI patient (female, paraplegic, AIS grade of $\mathrm{C}$ and with scan finding of subdural haemorrhage on T4 level). D: Schematic drawing of the lesion on the cord for analysing the lesion parameters, $\mathrm{DB}=$ dorsal midsagittal tissue bridges; $\mathrm{LA}=$ lesion area; $\mathrm{LL}=$ lesion length; $\mathrm{LW}=$ lesion width; $\mathrm{VB}=$ ventral midsagittal tissue bridges.
Comment [WM(57]: Please provide copy of permission letter, please add reference to the references list

Images in column B are a little blurry. Text is not editable. Do you have high resolution pictures for column $B$ ?

Please provide a figure where we can edit the text

Comment [MS58]: Ad 1) We now provide the permission letters for all required Figures

Ad 2) Unfortunately the authors did not get back to us and we cannot provide a Figure with a higher resolution

Comment [WM(59]: define Comment [MS60]: it is defined now Comment [WM(61]: how many months after surgery?

Comment [MS62]: 32.5 hours post operation

Comment [WM(63]: Please provide copy of permission letter; please add reference to the references list

We would need an editable part A, so lines and text can be changed to Lancet style

Comment [MS64]: Now provided. Comment [WM(65]: Copy of permission letter needed? Or also from Bizhan?

Comment [WM(66]: Copy of permission letter needed? Or also from Bizhan?

Comment [MS67]: The permission letter are uploaded. 
Figure 3: The lesion evolution over time Please provide figure title

Overview of the lesion evolution with persisting midsagittal tissue bridges over 1-year postinjury. Longitudinal T2-weighted sagittal MRI scans showing the evolution of the cervical lesion epicenter from a 27 year-old27-year-old complete SCI patient (male, tetraplegic, AIS grade A) in acute (one day post -SCI), subacute (1 month post-SCI) and chronic (12 months post-SCI)-phase after injury.

Figure 4: Lschematic representation of connections between qMRI methods and the neocortical microstructural features Please provide figure title

Relationships between different quantitative MRI readouts and the microstructural features reported previously in post mortem brain tissue ${ }^{97,98}$ - to which they have been linked. A coloured line between a quantitative MRI readout and a microstructural feature implies that this readout has been empirical linked to this feature. The relationships between MR contrast and microstructural features makes microstructural mapping through the combination of complementary quantitative MR images possible. MT = magnetisation transfer saturation; $\mathrm{PD}=$ proton density, $\mathrm{R} 1=$ longitudinal relaxation rate, $\mathrm{R} 2 *=$ effective transverse relaxation rate, $\mathrm{QSM}=$ quantitative susceptibility mapping, $\mathrm{R} 2=$ transverse relaxation rate, $\mathrm{dMRI}=$ diffusion weighted MRI. (Reprinted from Edwards et al. $\left(2018_{-}^{57}\right.$ ) under the terms of the CC-BY 4.0 license

(https://creativecommons.org/licenses/by/4.0/)).

Figure 5: Schematic representation of primary and secondary degenerative processes occurring remotely, above and below the injury site Please provide figure title

Primary damage at the focal epicenter of the lesion occurring within hours after injury $\mathbf{A}$, and secondary systematic degenerative processes occur remotely and with a certain time lag [A: define certain time $\operatorname{lag} \_B$, above, below and at the the primary injury site. Sensory and motor tracts affected by the injury undergo anterograde and/or retrograde (depending on the location) axonal degeneration and accompanying demyelination. ${ }^{6,12,64}$ - Lesion site shows the macrostructural evidence of primary intramedullary damage (e.g. oedema \&; haemorrhage?) and secondary changes such as post-traumatic cyst cavities as well asand spared tissue bridges $\llbracket$ A: possible to show in the figure? $]^{42,44}$ In the lumbar cord, the lower motor neurons located in the ventral horn may undergo trans-synaptic degeneration due to the loss
Comment [WM(68]: Editable text would be useful

Would some arrows help to highlight certain things?

Comment [MS69]: The new figure is editable and the arrows are added to the figure now to show the lesion epicenter area.

Formatted: Font: 12 pt, Bold

Comment [WM(70]: At time period for these phases

Comment [WM(71]: All parts of this need to be editable. The images of iron and neuronal fibres are a bit blurry.

Please also provide bar scale for all images

Comment [MS72]: This figure was taken from published material, and the scales are added to those figures authors provided us, the rest of the figures don't have a scale bar and authors did not provide a better resolution figure.

Formatted: Font: 12 pt, Bold

Formatted: Font: 12 pt, Font color: Red

Comment [WM(73]: Please add reference to the references list

Comment [MS74]: Reference has been added to the reference list.

Comment [WM(75]: This figure isn't editable.

Comment [MS76]: Now we uploade the ai file which are editable.

Formatted: Line spacing: 1.5 lines

Formatted: Font: 12 pt, Bold

Formatted: Font: 12 pt, Font color:

Red

Comment [MS77]: Now it is added the figure.

Comment [WM(78]: Please label on figure to help readers

Comment [MS79]: Now it is added 
of input from the injured corticospinal tracts. ${ }^{48}$ Similarly, second-order sensory neurons of the spinothalamic and dorsal column medial lemniscus systems can also be affected by transsynaptic degeneration. At the brain level, atrophic changes are located within the brainstem, ${ }^{51}$ cranial corticospinal tracts, primary motor cortices, insula, anterior cingulate cortex, and thalamus. ${ }^{7,8,14}$ Some of these areas (e.g. cortical and subcortical regions) present also with changes in the-myelin and iron content which is suggestive of demyelination and iron accumulation. ${ }^{9}$ [A: add a sentence on the illustrated MRI measures; "c" is very difficult to see]

DTI: diffusion tensor imaging, MPM: multi--parameter mapping, T1w and T2*w MRI: T1 weighted and $\mathrm{T} 2 *$ weighted magnetic resonance imaging,-Add abbreviations for T1w MRI, $\underline{\text { etc }}$
Comment [WM(80]: Please label on figure to help readers

Comment [MS81]: Now it is added

Comment [WM(82]: Label says" bilateral" but it's not consistency illustrated on both sides

Comment [MS83]: "bilateral" is removed now

Formatted: Font: 12 pt, Not Highligh Comment [WM(84]: Please be more specific and add supporting references.

Comment [MS85]: This is added now

The font color of " $\mathrm{C}$ " is changed now.

Comment [MS86]: The abbreviation are now added. 
Table 1: Completed clinical trials in spinal cord injury (SCI) using MRI as an outcome measure within the last 5 years
Formatted: Font: $12 \mathrm{pt}$, Bold

Formatted: Font: $12 \mathrm{pt}$, Bold

Formatted: Font: $12 \mathrm{pt}$, Bold

Formatted: Font: 12 pt, Bold

\begin{tabular}{|c|c|c|c|c|c|c|c|}
\hline \multirow{2}{*}{$\begin{array}{l}\text { Clinical } \\
\text { trials.gov } \\
\text { identifier }\end{array}$} & \multirow[t]{2}{*}{ Study Title } & \multirow[t]{2}{*}{ Intervention } & \multirow{2}{*}{$\begin{array}{c}\text { Enrollment (number } \\
\text { of } \\
\text { Participants)/Condit } \\
\text { ion }\end{array}$} & \multicolumn{2}{|c|}{ MRI as outcome measures } & \multirow{2}{*}{ MRI techniques } & \multirow{2}{*}{$\underset{\text { Status }}{4}$} \\
\hline & & & & $\begin{array}{l}\text { Primary } \\
\text { outcome }\end{array}$ & $\begin{array}{c}\text { Secondary } \\
\text { outcome }\end{array}$ & & \\
\hline NCT01325103 & $\begin{array}{l}\text { Evaluation of } \\
\text { Autologous } \\
\text { Mesenchymal } \\
\text { Stem Cell } \\
\text { Transplantation in } \\
\text { Chronic Spinal } \\
\text { Cord Injury: }\end{array}$ & $\begin{array}{l}\text { Autologous } \\
\text { Mesenchymal Stem } \\
\text { Cell }\end{array}$ & $\begin{array}{l}14 \text { Participants } \\
\text { Echronic spinal cord } \\
\text { injury }\end{array}$ & & $\begin{array}{l}\square \text { Lesion } \\
\text { volume }\end{array}$ & $\begin{array}{l}\text { Anatomical } \\
\text { Conventional MRI } \\
\text { scan (T1/T2 } \\
\text { weighted_MRI) } \\
\text { Stem Cell Res Ther. } \\
\text { 2014 Nor } \\
\text { 17:5(6):126. }\end{array}$ & $\begin{array}{l}\text { Completed } \\
\text { December } \\
20124 \\
\square \text { Published } \\
\text { results } \\
\end{array}$ \\
\hline NCT01624779 & $\begin{array}{l}\text { Intrathecal } \\
\text { Transplantation of } \\
\text { Autologous } \\
\text { Adipose Tissue } \\
\text { Derived MSC in } \\
\text { the Patients With } \\
\text { Spinal Cord } \\
\text { Injury }\end{array}$ & $\begin{array}{l}\text { Autologous adipose } \\
\text { tissue derived } \\
\text { mesenchymal stem } \\
\text { cells }\end{array}$ & $\begin{array}{l}15 \text { Participants } \\
\text { cEhronic spinal cord } \\
\text { injury }\end{array}$ & $\begin{array}{l}\square \text { Qualitative } \\
\text { lesion } \\
\text { assessment }\end{array}$ & & $\begin{array}{l}\text { Anatomical } \\
\text { Conventional MRI } \\
\text { scan (T1/T2 } \\
\text { weighted axial and } \\
\text { sagittal MRI) J } \\
\text { Spinal Cord Med. } \\
\text { 2016 Nov; } 39(6): \\
\text { 655-664. }\end{array}$ & $\begin{array}{l}\text { Completed } \\
\text { May } 2014 \\
\\
\square \text { Published } \\
\text { results }^{73}\end{array}$ \\
\hline NCT01739023 & $\begin{array}{l}\text { Safety of } \\
\text { Autologous } \\
\text { Human Schwann } \\
\text { Cells (ahSC) in } \\
\text { Subjects wWith } \\
\text { Subacute SCI }\end{array}$ & $\begin{array}{l}\text { Autologous Human } \\
\text { Schwann Cell }\end{array}$ & $\begin{array}{l}9 \text { Participants } \\
\text { Subacute SCI ( } 30 \\
\text { day-post injury) }\end{array}$ & & $\begin{array}{l}\nabla \text { Lesion } \\
\text { volume }\end{array}$ & $\begin{array}{l}\text { Anatomical } \\
\text { Conventional MRI } \\
\text { scan }(\mathrm{T} 1 / \mathrm{T} 2 \\
\text { weighted) seans-MRI } \\
\text { on the lesion area } \\
\text { JOURNAL OF } \\
\text { NEUROTRAUMA } \\
\text { 34:2950 2963 }\end{array}$ & $\begin{array}{l}\text { Completed } \\
\text { Auqust } 2016 \\
\text { September } 2017 \\
\square \text { Published } \\
\text { results }^{75}\end{array}$ \\
\hline
\end{tabular}

Remove column "exploratory":

it is removed now.

Meaning of the column "MRI details" is unclear - why is there a reference?

The column title changed to MRI techniques, but in the first inquiry of the paper by reviewer group, it was strongly suggested to provide references on the list of clinical trials. Now The references are added to the review citation list as well.

Column "Status Primary Date": please add if results have been published or not; The results are published in the cited papers in the table.

also check if it matches Clinical trials.gov, for the last trial, for example, is say completed in Aug 2016 but you mentioned Sep 2017.

It is corrected now.

Might be useful if you could add any caveats/things to consider/clinical implications:

We could not find any complications reported in the published material regarding MRI technique applied in the clinical trials listed here.

Provide table title: it is added now.

Formatted: Font: 10 pt, Bold 
Table2: Different MRI techniques applied in SCI and their with corresponding outcome measures

the MRI technique listed from conventional MRI (e.g. T1 and T2 weighted MRI) to more advanced MRI methods such as diffusion tensor imaging (DTI), Multi parameter mapping (MPM) and Functional MRI (fMRI) of brain and spinal cord all-deseribed in the text.

\begin{tabular}{|c|c|}
\hline MRI techniques & Outcome measures \\
\hline $\begin{array}{l}\text { Sagittal and axial conventional T2-weigthed } \\
\text { MRI of the spinal cord at the injury level }\end{array}$ & $\begin{array}{l}\text { - } \text { extent of the intramedullary injury \& lesion length }{ }^{27,28,38} \\
\text { - } \text { haemorrhage }^{34} \\
\text { - } \text { oedema }^{36} \\
\text { - } \text { spinal cord swelling compression }^{99}\end{array}$ \\
\hline $\begin{array}{l}\text { Sagittal conventional T2-weigthed MRI of the } \\
\text { spinal cord at the injury level }\end{array}$ & - $\quad$ preserved midsagittal tissue bridges ${ }_{4}^{42,44}$ \\
\hline $\begin{array}{l}\text { T1-weighted MRI (above the injury level, } \\
\text { cervical cord and brain) }\end{array}$ & - $\quad$ cervical cord and brain atrophy ${ }^{7,8,10,46,49,52}$ \\
\hline $\begin{array}{l}\text { T2*-weighted MRI of the cervical and lumbar } \\
\text { cord (remote from the injury site) }\end{array}$ & - $\quad$ grey and white matter atrophy of the spinal cord ${ }^{6,48}$ \\
\hline $\begin{array}{l}\text { Diffusion tensor imaging (DTI) of the cord } \\
\text { and brain } \\
\text { (remote from the injury site) }\end{array}$ & - $\quad$ axonal degeneration \& demyelination ${ }^{6,11,12,14,64}$ \\
\hline $\begin{array}{l}\text { Multi parameter mapping (MPM) of the } \\
\text { cervical cord and brain (above the injury } \\
\text { level) }\end{array}$ & - $\quad$ demyelination $\&$ iron deposition ${ }^{7-10}$ \\
\hline $\begin{array}{l}\text { Functional MRI (fMRI) of brain and spinal } \\
\text { cord (remote from injury level) }\end{array}$ & - $\quad$ functional networks \& plasticity in brain ${ }^{19-21} \&$ spinal cord ${ }^{22-25}$ \\
\hline
\end{tabular}

Formatted: Font: 12 pt, Bold

Formatted: Font: Bold

Formatted: Font: 12 pt, Bold

Formatted: Font: Bold

\section{Formatted Table}

Field Code Changed

Not clear why these references have been listed here- are they refereeing to a particular outcome measure if yes, please add to the corresponding outcome measure.

The references are refereeing to a particular outcome measure and now it is changed as requested.

Please add all references to the reference list;

They are added now.

References should be in the Vancouver style and numbered in the order in which they first appear in the manuscript. If the references "move" from the body text into tables or figures, please maintain the sequence of citation

The changes are applied as requested.

Provide table title and abbreviation legend; why are the techniques ordered in this way - please make the rational clear in the text or legend;

Title is added,

the techniques are ordered from conventional MRI to qMRI as it is-mentioned in the main text. 


\section{$\underline{\text { Panel }}$}

\section{Clinical and electrophysiological assessments}

To date, the symptoms and signs of myelopathy (i.e., the degree of sensorimotor deficit and the emergence of neuropathic pain) are assessed clinically and by electrophysiological tests. The current gold standard in assessing clinical impairment is by means of the International $\underline{\text { Standards for the Neurological Classification of Spinal Cord Injury (ISNCSCI) protocol. }}{ }^{1}$ This test is routinely performed at admission by a qualified clinician who tests key muscles for strength and all dermatomes for light-touch and pin-prick sensation. A score is then calculated which is used to classify patients' overall impairment into a scale with five ranked categories (ASIA impairment scale) - A to E. Category A is the most severely damaged spinal cord with no motor and sensory function below the level of injury whereas category $\mathrm{E}$ features no clinically relevant impairment. Although defining AIS categories is a fairly easy process, it does not capture the entire extent of primary and secondary injury mechanisms. Consequently, this leads to considerable heterogeneity within an AIS category, which limits its applicability as a surrogate endpoint in clinical trials. Thus, large clinical trials are needed to distinguish a treatment effect from natural history. To address this drawback, dedicated prediction models (i.e., unbiased recursive partitioning) have recently been established. ${ }^{2-4}$ These models aim to reduce the heterogeneity within SCI cohorts thereby improving patient stratification.

$\underline{\text { Recently, more refined measures have also been developed. For upper limb function, manual }}$ dexterity is assessed by the Graded Redefined Assessment of Strength Sensibility and Prehension (GRASSP) score ${ }^{5}$, functional independence by the functional independence (SCIM) score ${ }^{6}$, and neuropathic pain intensity is commonly assessed with pain 
questionnaires. ${ }^{7}$ Neurophysiological recordings such as motor and sensory evoked potentials ${ }^{8}$, as well as contact heat evoked potentials ${ }^{9}$, can provide additional information about the integrity of impulse conductance of motor and distinct sensory pathways. ${ }^{10}$ However, these examinations report on impaired function related to focal injury; they do not reflect remote neurodegeneration and functional reorganizational processes that occur with distinct (delayed) temporal profiles. ${ }^{11}$ However, all these examinations fail to differentiate or elucidate the mechanisms responsible for recovery processes in-vivo.

1 Kirshblum S, Waring W. Updates for the International Standards for Neurological Classification of Spinal Cord Injury. Phys Med Rehabil Clin N Am 2014; 25: 505-17, vii.

2 Tanadini LG, Hothorn T, Jones LAT, et al. Toward Inclusive Trial Protocols in Heterogeneous Neurological Disorders: Prediction-Based Stratification of Participants With Incomplete Cervical Spinal Cord Injury. Neurorehabil Neural Repair 2015; 29: $\underline{867-77 .}$

3 Tanadini LG, Steeves JD, Hothorn T, et al. Identifying Homogeneous Subgroups in Neurological Disorders: Unbiased Recursive Partitioning in Cervical Complete Spinal Cord Injury. Neurorehabil Neural Repair 2014; 28: 507-15.

4 Velstra I-M, Bolliger M, Tanadini LG, et al. Prediction and Stratification of Upper Limb Function and Self-Care in Acute Cervical Spinal Cord Injury With the Graded Redefined Assessment of Strength, Sensibility, and Prehension (GRASSP). Neurorehabil Neural Repair 2014; 28: 632-42.

5 Velstra I-M, Curt A, Frotzler A, et al. Changes in Strength, Sensation, and Prehension in Acute Cervical Spinal Cord Injury. Neurorehabil Neural Repair 2015; 29: 755-66.

$6 \quad$ Itzkovich M, Shefler H, Front L, et al. SCIM III (Spinal Cord Independence Measure version III): reliability of assessment by interview and comparison with assessment by observation. Spinal Cord 2018; 56: 46-51.

$7 \quad$ Hatch MN, Cushing TR, Carlson GD, Chang EY. Neuropathic pain and SCI: Identification and treatment strategies in the 21st century. J Neurol Sci 2018; 384: 75 83.

8 Petersen JA, Spiess M, Curt A, et al. Upper Limb Recovery in Spinal Cord Injury: Involvement of Central and Peripheral Motor Pathways. Neurorehabil Neural Repair 2017; 31: 432-41.

9 Jutzeler CR, Ulrich A, Huber B, Rosner J, Kramer JLK, Curt A. Improved Diagnosis of Cervical Spondylotic Myelopathy with Contact Heat Evoked Potentials. $J$ Neurotrauma 2017; 34: 2045-53.

10 Hupp M, Pavese C, Bachmann LM, Koller R, Schubert M. Electrophysiological Multimodal Assessments Improve Outcome Prediction in Traumatic Cervical Spinal Cord Injury. J Neurotrauma 2018; : neu.2017.5576.

11 Freund P, Friston K, Thompson AJAJ, et al. Embodied neurology: an integrative framework for neurological disorders. Brain 2016; 139: 1855-61. 

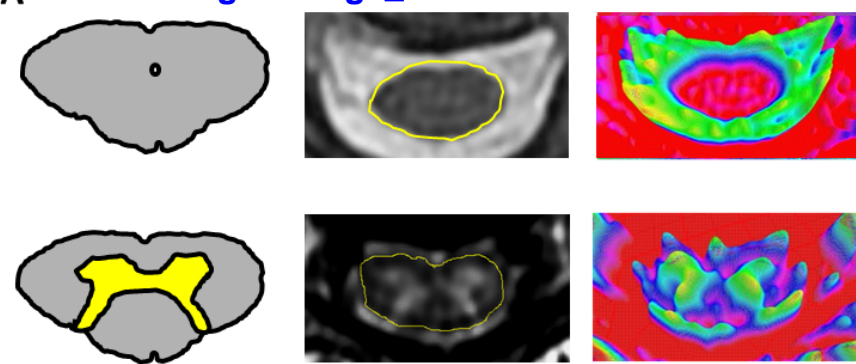

BASIC 1: Intramedullary T2 hyperintensity is approximately confined to central gray matter.
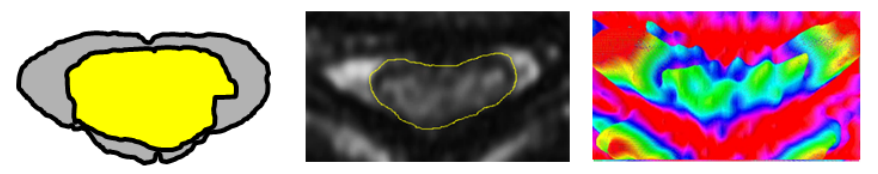

BASIC 2: Intramedullary T2 hyperintensity extends beyond expected gray matter margins to involve spinal white matter, but does not involve entire transverse extent of the spinal cord.
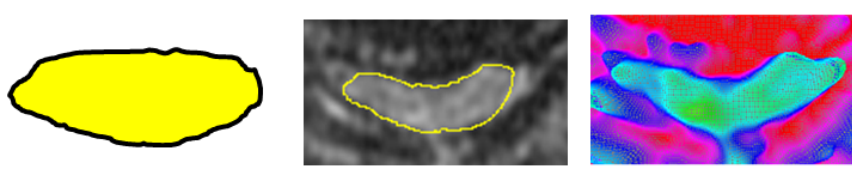

BASIC 3: Intramedullary T2 hyperintensity involves entire transverse extent of spinal cord.
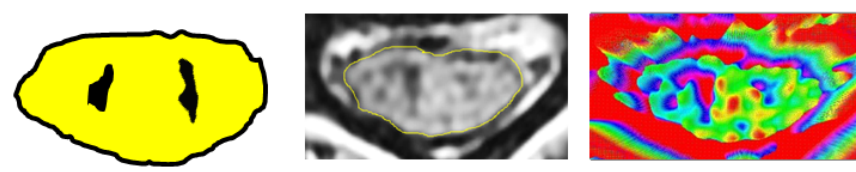

BASIC 4: Grade 3 injury plus discrete T2 hypointense foci, consistent with

macrohemorrhage.

BASIC 0: No appreciable intramedullary cord signal abnormality. 
Figure 2

Click here to download Figure: Fig2_lesion_segmentation.ai
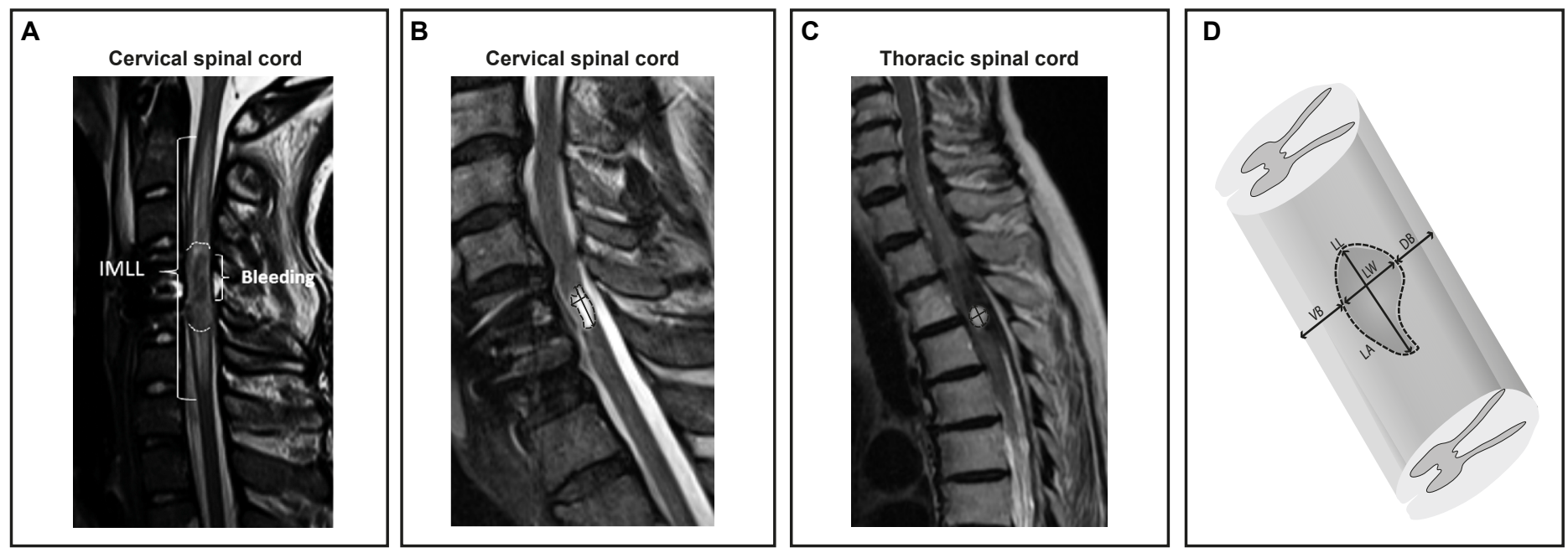


\section{Figure 4}

Click here to download Figure: Fig4_qMRI_methods.ai

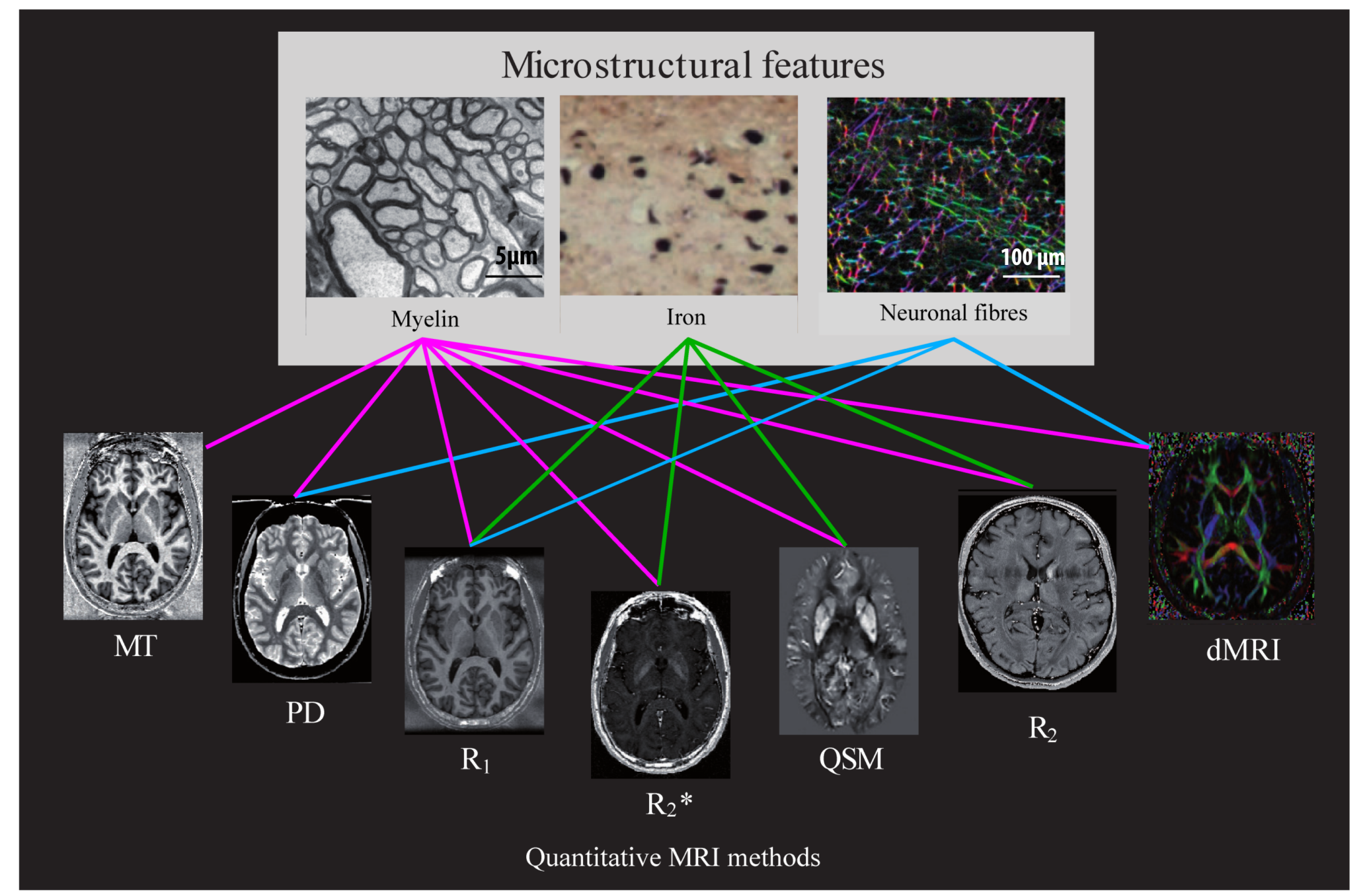




\section{Point2point reply}

\section{Editorial comments:}

Additional editorial points:

E1. In same instances, the clinic implications are missing or could be made more obvious.

Now in the paragraph entitled "Implication for clinical trials" we added: "The primary endpoint of choice in SCl trials so far is an improvement in clinical outcome measures. However, neuroimaging biomarkers, hold potential to supplement these clinical measures as they are sensitive to neuronal changes even when they do not yet translate into obvious clinical benefits."

E2. No panel providing an overview over the various MRI techniques has been provided as indicated in the pointby-point response.

We have included a panel that describes the gold standard clinical and electrophysiological assessments as well as a Table (Table 2) which outlines the various MRI techniques, their outcome measures and the references where presented.

E.3 ICMJE forms: they are still not match the declarations made in the text; please submit updated forms

The forms are now updated and submitted once more as requested.

E4. You mentioned "Moreover, we now have added a second table presenting evidence about the sensitivity of conventional and qMRI sequences to reveal specific structural changes and hence could be used as a potential outcome measure." I can't find these sensitive measures in either of these tables.

We added the second table in response to the reviewer 2.2 comment during the former revision process. As requested we present the outcome measures in the table, however, we do not feel comfortable to make a statement of the sensitivity at this point.

E5. Not all responses mentioned in the point-by-point response can be found in the clean manuscript version, eg, "We agree with this reviewer and have now added to the section 'Implication for clinical trials' the following: "Despite a therapeutic intervention having an effect on imaging outcome such as halting atrophy, there is still some disconnect between changes in imaging outcome and clinically meaningful recovery; the ultimate goal of a successful clinical trial. Thus, it may be useful to employ more than one imaging outcome in future trials to maximize understanding and interpretation of clinically meaningful findings 85."

We now added the paragraph mentioned above in the text as well as added a panel and a Table (Table 2).

Please go carefully over the previous reviewer comments and integrate the changes.

We went over the reviewer's comments and integrated all changes as requested. The abstract is slightly changed accordingly. One reference suggested by Reviewer\#02 (Aarabi B et al 2018 Neurotrauma) was not added previously, but now it is in the reference list.

\section{Reviewers' comments:}

Reviewer \#2: Dear authors:

R2.1-Page 2 line 5 change osseoligamentous spinal elements to discoligamentous complex.

Now changed.

R2.2-Page 3, line 3 change disco-ligamentary to discoligamentous complex.

Now changed.

R2.3-Page 16 paragraph 2, line 10 there is a typo.

The phrase with the typo has been deleted. 
R2.4-Paragraph 3 line 1 in page 16 neuraxis.

This has been changed throughout the text.

R2.5-Page 17 first paragraph line 4 add neurosurgical.

Now added.

R2.6-Page 17 line 15 QMRI.

We prefer to keep qMRI as it is the convention.

R2.6-Page 21, Ref 5 typo (AJ). Line

Now corrected.

R2.7-Page 22 Ref 46 needs more info about the proceedings.

This reference has been replaced throughout the text with the full journal paper (David et al 2019 Neurology) recently published.

R2.8-Page 22 ref 48 check PubMed

We checked the reference 48: Chen $Q$, Zheng W, Chen X, et al. Brain Gray Matter Atrophy after Spinal Cord Injury: A Voxel-Based Morphometry Study. Front Hum Neurosci 2017; 11: 211.

on PubMed. It is the same reference we would like to cite in our review.

R2.9-Check ref 51.

We checked the reference 51 and it is correctly cited: Seif M, Ziegler G, Freund P. Progressive ventricles enlargement and CSF volume increases as a marker of neurodegeneration in $\mathrm{SCl}$ patients: $\mathrm{A}$ longitudinal MRI study. J Neurotrauma 2018; : neu.2017.5522.

R2.9-Page 24 check ref 77 for validity.

The paper is not published and therefore we would like to make reference to the peer reviewd abstract.

Seif M, Leutritz T, Samson RS, et al. A multi-center study on fast full-brain quantitative multi-parameter mapping of R1, MT, and R2*: scan-rescan repeatability and inter-site reproducibility. In: ISMRM. , 2018: 1119.

R2.10-Page 24 ref 83 cut the typo.

Now corrected.

Reviewer \#3: Overall, the authors have responded well to the critiques with extensive revisions of their manuscript.

I suggest two small revisions:

R3.1- in the section "Implication for clinical trials", there should be explicit acknowledgement that clinical outcome is invariably the outcome of choice in SCI trials, that surrogate markers such as MRI, no matter how advanced, will never substitute for clinical outcome. The way that this section is now written is misleading in overemphasizing the importance of bioimaging markers.

We now open this paragraph with the following sentence: "The primary endpoint of choice in SCl trials so far is an improvement in clinical outcome measures. However, neuroimaging biomarkers, hold potential to supplement these clinical measures as they are sensitive to neuronal changes even when they do not yet translate into obvious clinical benefits." 
Reviewer \#4: This review summarizes the application of conventional and advanced MRI techniques to study spinal cord injury (SCl). The main findings of studies using conventional MRI to reveal focal pathology and cord atrophy, as well as of studies applying quantitative MRI to track microstructural damage and functional reorganization are presented. The Review has been fully restructured and re-written compared to the previous version. The revised version is now much more informative and clearer in its subsections; the title has also been changed and is now much more appropriate. Still, there are some changes to the text to be performed:

R4.1 Paragraph entitled "Use of conventional MRI to reveal focal cord pathology - immediate changes at the epicentre". It is written that "recent spinal cord imaging studies [..] based on signal intensity changes of T2 and T1-weighted scans". Are the detected signal changes always hyperintensities on T2 and hypointensities on T1 scans? If so, please state it more explicitly. If not, please explain better which patterns of signal changes are detected in the different sequences.

We have now made this statement more explicitly: "By taking advantage of such techniques, spinal cord imaging studies have investigated primary changes (i.e. macrostructural) immediately following the injury, focally at the injury site and based on hyperintensity signal changes of sagittal and axial T2weighted and hypointensity signal changes of T1-weighted MRI scans ${ }^{31-36 . "}$

R4.2 Paragraph entitled "Conventional MRI: tracking remote cord and brain atrophy". It is written that "highresolution $\mathrm{T}^{*}$-weighted scans allow to segment grey and white matter of the cord". It would be more appropriate to substitute "T2*-weighted scans" with "multi-echo gradient echo scans".

\section{Now replaced.}

R4.3 Paragraph "Progressive brain atrophy". The first sentence states that spinal cord trauma induced brain atrophy in the primary motor cortex, anterior cingulate and several other brain regions. However, in the following sentence, it is stated that primary motor cortices and anterior cingulate cortex hypertrophy is associated with neuropathic pain. This is somewhat contradictory with the previous sentence. Are these regions atrophic or hypertrophic? This concept has to be better explained.

\section{We have now rewritten this section accordingly:}

"For example, greater volume reductions in the brainstem during the first 6 months post-SCl were associated with poorer recovery of lower limb motor function. Interestingly, performance improvements due to intensive lower limb training in chronic $\mathrm{SCl}$ patients lead to volume increases within the atrophied brainstem, indicating reorganizational processes (Villiger et al. 2015). Likewise neuropathic pain intensity has been shown to be associated with reductions in primary sensory cortices and thalamus ${ }^{47}$ as well as increases in grey matter volume within the anterior cingulate gyrus and primary motor cortices 52 ."

R4.4 Paragraph "clinical qMRI studies". Please substitute "The advent of a spinal cord template, the spinal cord toolbox and post processing tools now offers" .. with "The advent of a spinal cord template and postprocessing tools included in the spinal cord toolbox now offers.".

Now adapted.

R4.5 I would use the following title "Predicting clinical outcomes with qMRI" instead of "Predicting outcome with MRI"

Now changed to "Predicting outcome" as suggested by the editor.

R4.6 In the paragraph entitled "Implications for clinical trials", when describing feasibility studies on multicentre DTI data, it is appropriate to include the study of Samson et al (Plos One 2016), which is focused specifically on DTI data of the spinal cord.

We now included this reference. 